\title{
A Modular Neural Network Decision Support System in EMG Diagnosis
}

\author{
C.I. Christodoulou ${ }^{1,3}$, C.S. Pattichis ${ }^{2}$, W.F. Fincham ${ }^{1}$ \\ ${ }^{1}$ Department of Electronic Engineering, Queen Mary and Westfield College, \\ University of London, Mile End Road, London EI 4NS, U.K. \\ e-mail:W.F.Fincham@qmw.ac.uk \\ ${ }^{2}$ Department of Computer Science, University of Cyprus, 75 Kallipoleos. Str., \\ P.O.Box 578, 1678 Nicosia, Cyprus. \\ e-mail: \{cschr2, pattichi\}@turing.cs.ucy.ac.cy \\ ${ }^{3}$ Cyprus Institute of Neurology and Genetics, P.O.Box 34.62, \\ 1683 Nicosia, Cyprus
}

\begin{abstract}
Motor unit action potentials (MUAPs) recorded during routine electromyographic (EMG) examination provide important information for the assessment of neuromuscular disorders. The objective of this study was to design, develop, and test a decision support system which mimics the decision making process carried out by the expert neurophysiologist in MUAP analysis where: (i) the statistics of MUAP features are compared to normal reference values, and (ii) the individual MUAP waveforms are visually evaluated in sequence. The system consisted of the following two modular neural network subsystems. In the first subsystem, the statistics for each subject of multiple features extracted from the MUAP waveforms were fed into multiple classifiers, and the classification results were combined in order to improve the diagnostic yield. The feature sets computed, were: (i) the time domain parameters, (ii) the frequency domain parameters, (iii) the autoregressive coefficients, (iv) the cepstral coefficients and (v) the wavelet transform coefficients. The classifiers implemented were: (i) the back-propagation (BP), (ii) the radial basis function (RBF) network and (iii) the self-organising feature map
\end{abstract}


(SOFM). In the second subsystem, the individual MUAPs obtained by a subject were fed sequentially into the classifier and the classification results were combined. For this subsystem the time domain parameters, the MUAP waveforms, and the SOFM classifier were used. The outputs of the two subsystems were further combined in order to obtain the overall diagnostic yield. The proposed system was developed for the assessment of normal subjects and subjects suffering with myopathy and motor neuron disease. It was shown that the modular neural networks system enhanced the diagnostic performance of the individual classifiers making the whole system more robust and reliable.

Keywords: Modular Neural Networks, Decision Support Systems, MUAP, EMG.

\section{Introduction}

Recent advances in computer aided technology in health care make a vast amount of complex medical information available to the physician, which is almost impossible to be processed manually in an efficient way. Therefore, computerised decision support systems are highly recommended for a more efficient, reliable, uniform, and fast processing of the available data. Such systems are not intended to replace the physician but rather to provide a second unbiased opinion which will assist the physician in making a more accurate disease assessment and diagnosis (France and Santucci, 1991). The development of decision support systems based on artificial neural networks (ANN) has shown promising results in different areas of medical applications (Galant, 1995). The motivation behind the use of ANN lies in their capacity for making no assumptions about the underlying probability density functions, finding near-optimum solutions from incomplete data sets, and the fact that learning is accomplished through training. In addition to these characteristics, neural networks can combine data of a different nature in one system, such as data derived from clinical 
and W.F. Fincham

protocols and laboratory data obtained from measurements and features from signals and images, thus forming an integrated diagnostic system (Schizas $e t$ al., 1994). The performance of ANN models can be further enhanced by the development of modular neural networks, where the outputs of different modular neural modules are combined in order to form the final output of the system. Modular neural networks easily allow the combined use of supervised and unsupervised learning paradigms or different learning algorithms. This combines the advantages of the individual modules and can improve the overall performance. The objective of this study is to investigate the usefulness of modular neural networks in the development of a decision support system for neuromuscular disorders based on electromyographic (EMG) findings.

The diagnosis of neuromuscular disorders is a naturally complex and difficult problem to investigate. This group of disorders affects the brain and spinal cord, nerves, or muscles. Their main characteristic is muscular weakness and/or wasting, with the life expectancy of many sufferers being considerably reduced. Early detection and diagnosis of these diseases by clinical examination and laboratory tests are essential for their prognosis, management, and prevention. Laboratory tests include: (i) neurophysiological examinations, like EMG and nerve conduction studies, (ii) interpretation of human muscle biopsy i.e. image analysis, (iii) genetic and molecular genetic assessment, and (iv) biochemical assessment. In our study, the problem of computer aided EMG decision making is investigated. Computer aided EMG decision making is very important because it allows a standardized, more accurate, and less time-consuming assessment of EMG findings.

The shapes of motor unit action potentials (MUAPs) composing the EMG signal, provide an important source of information for the assessment of neuromuscular disorders. In computer aided EMG the MUAP waveforms derived by quantitative EMG techniques (Stalberg et al., 1986), and their computed parameters are presented to the physician in order to assist him in the assessment of the disease and subsequently in reaching a final diagnosis. The experienced neurophysiologist decides on the disease class by visually 
examining the shape of the MUAP waveforms in conjunction with comparison of the time domain parameters with reference values (Rosenfalck, 1975). A computerised decision support system which will automatically evaluate all of the above given data in a consistent and uniform marmer, and which will give the physician a second, unbiased opinion will provide an important aid in the current practice of clinical neurophysiology.

The objective of this study was to design, develop, and test a decision support system which tries to mimic the decision making tasks carried out by the expert neurophysiologist where: (i) the statistics of MUAP features are compared to normal reference values, and (ii) the individual MUAP waveforms are visually evaluated in sequence. The system consisted of two modular neural network subsystems. In the first subsystem, the statistics for each subject of multiple features extracted from the MUAP waveforms were fed into multiple classifiers, and the classification results were combined in order to improve the diagnostic yield. The feature sets extracted, were: (i) the time domain parameters, (ii) the frequency domain parameters, (iii) the autoregressive coefficients, (iv) the cepstral coefficients and (v) the wavelet transform coefficients. The classifiers implemented were: (i) the error back-propagation (BP), (ii) the radial basis function (RBF) network and (iii) the self-organising feature map (SOFM). In the second subsystem, the individual MUAPs obtained by a subject were fed sequentially into the classifier and the classification results were combined. For this subsystem the time domain parameters, the MUAP waveforms, and the SOFM classifier were used. The outputs of the two subsystems were further combined in order to obtain the overall diagnostic yield. The proposed modular neural network system was developed for the assessment of normal subjects and subjects suffering with myopathy and motor neuron disease. It was shown that the modular neural networks system enhanced the diagnostic performance of the individual ANN classifiers making the whole system more robust and reliable. 
and W.F. Fincham

\subsection{A Brief Survey of Previous Work in EMG Diagnosis}

A number of quantitative EMG techniques were developed by different researchers during the last two decades (Stalberg et al., 1986) providing the physician with valuable information. However, in order to facilitate the efficient processing of all the available information, including the assessment of disease, and the final diagnosis, the need had emerged for integrated, automated decision making systems. Towards this direction, different methodologies have been followed. Blinowska et al. (1980) proposed the use of discriminant analysis for the evaluation of MUAP findings, whereas Coatrieux et al. (1983) applied cluster analysis techniques for the automatic diagnosis of pathology based on MUAP records. Furthermore, knowledge based systems were also used for the development of intelligent EMG diagnostic systems. MUNIN (Muscle and Nerve Inference Network) was developed by Andreassen and co-workers (1989) based on a casual probabilistic network for the classification of EMG findings. KANDID (Knowledge based Assistant for Neuromuscular Disorders Diagnosing) was developed by Fuglsang-Frederiksen and his group (1989), where knowledge was extracted from the human expert and formulated into logical rules interpretable by the computer. HINT (High Level Inferencing Tool) was developed by Schofield (1997) based on an anatomical network for the interpretation of neurophysiological studies of the peripheral nervous system. These systems were based on rules defined by human experts, trying to mimic the process an experienced neurophysiologist follows in making a diagnosis. However, this may cause some limitations to the systems because medical knowledge is difficult to be formalised in explicit rules, and also opinions of human experts may differ. Also the use of probability densities in the Bayesian approach assumes that the input data are statistically independent which often does not apply in diagnostic practice since several symptoms of the disease may have the same organic cause.

In another approach computational intelligence techniques, including artificial neural networks, genetic algorithms and fuzzy systems were used for the development of intelligent systems. In these systems no experts have 
to define any rules but the rules are directly derived from the raw input data. Pattichis (1992) and Pattichis et al. (1995) used the averaged MUAP parameters as input in both supervised and unsupervised learning ANN models. Schizas et al. (1994) combined the above input data with clinical data and other laboratory data into an integrated diagnostic system based on the SOFM algorithm for unsupervised learning. Furthermore, Pattichis and Schizas (1996), also applied genetics-based machine learning (GBML) for the assessment of certain neuromuscular disorders. The diagnostic yield achieved by the above mentioned ANN and GBML models using the same input data set as in this work was of the order of $80 \%$. These findings are compared with the modular neural network system proposed in this study in the discussion section.

The paper is organised as follows. In section 2 the material and the EMG feature extraction are described. In section 3.1 the definition for modular neural networks is given and their properties are highlighted. In section 3.2 the first modular subsystem, using multiple features and multiple classifiers is described whereas in section 3.3 the second modular multi-MUAP classifier subsystem is presented. Section 4 covers the results of the system, and section 5 the discussion. In section 6, conclusions are given and appendix A covers in more detail the analysis for the extraction of the time domain, autoregressive, spectral, cepstral and wavelet transform features.

\section{Material and EMG Feature Extraction}

\subsection{Material}

Of the many neuromuscular disorders that have been clinically identified, two basic pathological processes have been found: muscle fibers are either lost through a degenerative process or there is loss of the motor neurons and their axons. When muscle fibers are lost, it is termed a myopathy, whereas when neurons or their axons are lost it is termed a neuropathy. From the large number of such disorders only two groups were considered in this study: myopathy (MYO), and motor neuron disease 
and W.F. Fincham

(MND). These two categories of MYO and MND were selected because the former is purely a disorder of the muscle fiber per se, while the latter is purely a disorder of the motor neuron. Myopathies are a group of diseases that affect primarily skeletal muscle fibers and they are divided into two groups, according to whether they are inherited or acquired. Most muscular dystrophies are hereditary and there are four main types, namely Duschenne's, Backers, fascioscapulohumeral, and limb girdle. These diseases show a progressive clinical course from birth or after a variable period of apparently normal infancy. One of the most frequently acquired myopathies is polymyositis, which is characterized by acute or subacute onset with muscle weakness progressing over a matter of weeks. MND is a disease causing selective degeneration of the upper and lower motor neuron. This disease affects middle to old aged people, with progressive widespread loss of motor neurons usually leading to death within three to five years. In the advanced stages of this disease, large motor units also denervate. The MYO and MND disorders cause structural reorganisation of the motor unit, the smallest functional unit of the muscle. In clinical neurophysiology, motor unit morphology can be studied by recording its electrical activity, the procedure known as EMG.

In this study, EMG was recorded from the biceps brachii muscle at a slight voluntary contraction for 5 seconds using the concentric needle electrode. The recordings points within the muscle were standardized, and MUAPs were recorded from three to five needle insertions. The electrode was usually advanced at least 3-5 $\mathrm{mm}$ into the muscle before recording. The electrode was also moved at least 3-5 mm between recordings to make sure that different MUAPs were recorded. The signal was then bandpass filtered at $3 \mathrm{~Hz}$ to $10 \mathrm{KHz}$, and sampled at $20 \mathrm{KHz}$ with 12 bits resolution. The signal was then lowpass filtered at $10 \mathrm{KHz}$. MUAP waveforms with similar shape were identified and selected from the EMG recording using an unsupervised learning neural network system (Christodoulou and Pattichis, 1995). Similar MUAPs were averaged to obtain the averaged MUAP waveform, which is thereafter simply referred to as MUAP. The MUAP epoch consisted of 512 samples (25.6 ms). In quantitative EMG studies it is 
appropriate to record 20 MUAPs from the muscle of each subject. This number is considered an acceptable sample of the whole muscle (Buchthal, 1957). The 20 MUAP waveforms and the statistics of their time domain parameters are evaluated by the neurophysiologist in order to assist him in the assessment of the disease and subsequently in reaching the final diagnosis.

A total of 800 MUAPs were recorded from 40 subjects, 12 normal (NOR), 13 MYO and 15 MND. Diagnostic criteria were based on clinical opinion, biochemical data and muscle biopsy. Only subjects with no history or signs of neuromuscular disorders were considered as normal. The modular neural network system was trained and tested for the three classes. Different sets with eight subjects from each group were randomly selected to form the ANN training set, whereas the remaining subjects formed the ANN evaluation set. The same data set was also used in the studies by Pattichis (1992) and Elia (1994).

\subsection{EMG Feature Extraction}

For each subject a matrix of 20 MUAP waveforms x 512 samples was available for further processing. From each MUAP waveform the following feature sets were extracted: (i) the time domain parameters, (ii) the frequency domain parameters, (iii) the autoregressive coefficients, (iv) the cepstral coefficients and (v) the wavelet transform coefficients. These features were computed for the NOR, MYO and MND groups presented in the previous section. A brief description of these feature sets is given in this section whereas a more detailed analysis of their computation and summary statistics are given in appendix A. Figure 1 shows 20 MUAP waveforms and their corresponding feature sets.

\subsubsection{Time domain parameters.}

Time domain parameters are the most widely used parameters in clinical neurophysiology for the interpretation of EMG findings. The following time domain parameters were computed from the MUAP waveforms as illustrated in Fig. 2 (Pattichis, 1992): duration, spike duration, amplitude, area, spike 


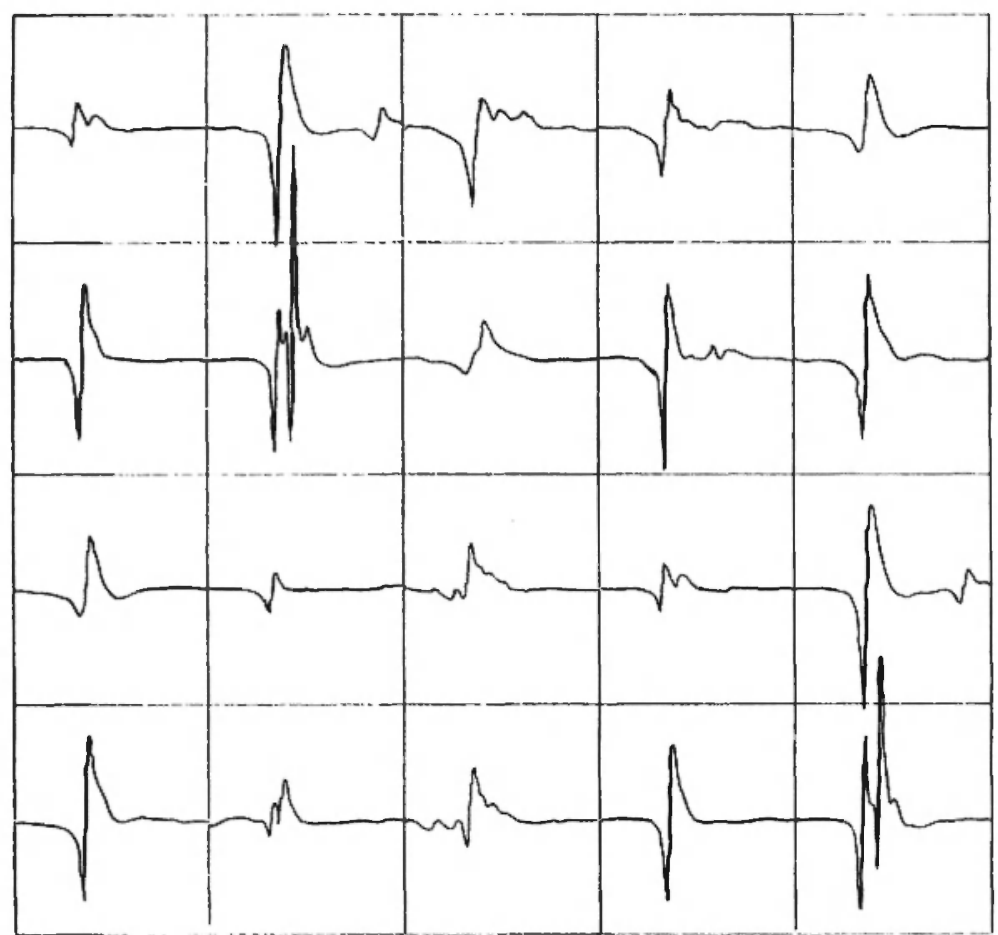

b) Average time domain parameters

\begin{tabular}{|c|c|c|c|c|c|c|}
\hline $\begin{array}{c}\text { Duration } \\
\boldsymbol{m s}\end{array}$ & $\begin{array}{c}\text { Spike } \\
\text { duration } \\
\boldsymbol{m s}\end{array}$ & $\begin{array}{c}\text { Amplitude } \\
\boldsymbol{m} \boldsymbol{V}\end{array}$ & $\begin{array}{c}\text { Area } \\
\boldsymbol{m V} \mathbf{m s}\end{array}$ & $\begin{array}{c}\text { Spike area } \\
\boldsymbol{m} \boldsymbol{V} \boldsymbol{m s}\end{array}$ & Phases & Turns \\
\hline 6.32 & 4.60 & 0.26 & 0.17 & 0.12 & 2.20 & 3.14 \\
\hline
\end{tabular}

c) Average frequency domain parameters

\begin{tabular}{|c|c|c|c|c|}
\hline $\begin{array}{c}F M E D \\
H z\end{array}$ & $Q$ & $\begin{array}{c}M_{0} \\
m V^{2}\end{array}$ & $\begin{array}{c}M_{l} \\
m V^{*} / s^{\star} 10^{3}\end{array}$ & $\begin{array}{c}M_{2} \\
m V^{2} / s^{2 *} 10^{6}\end{array}$ \\
\hline 758 & 0.553 & 6.2 & 4.53 & 5.78 \\
\hline
\end{tabular}

Fig. 1: An EMG study of a MYO subject. a) 20 MUAP waveforms in the time domain. b) Average time domain parameters. c) Average frequency domain parameters. 


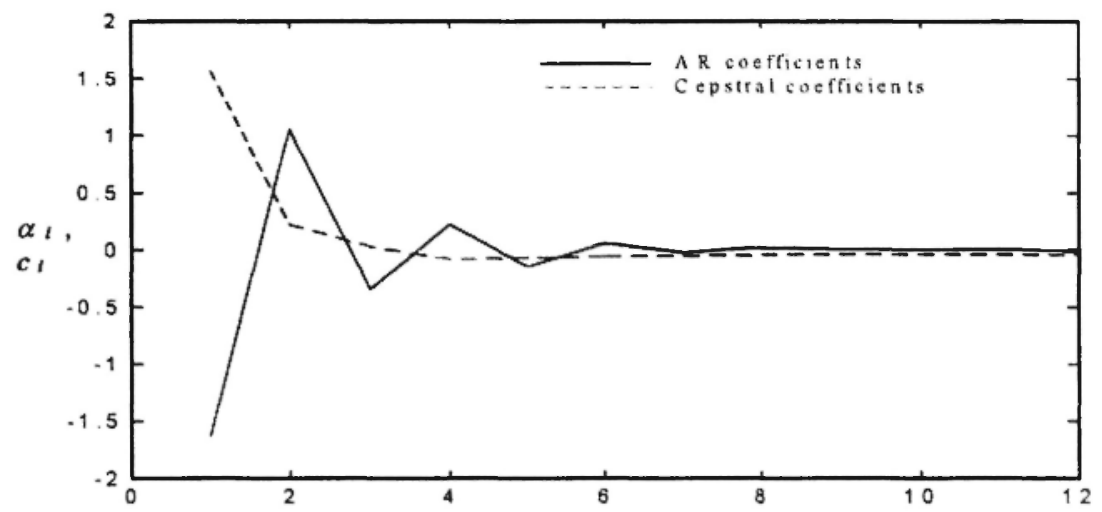

coefficient index

e) Average time-frequency plot of wavelet coefficients DAU4 (black=maximum power, white=minimum power)

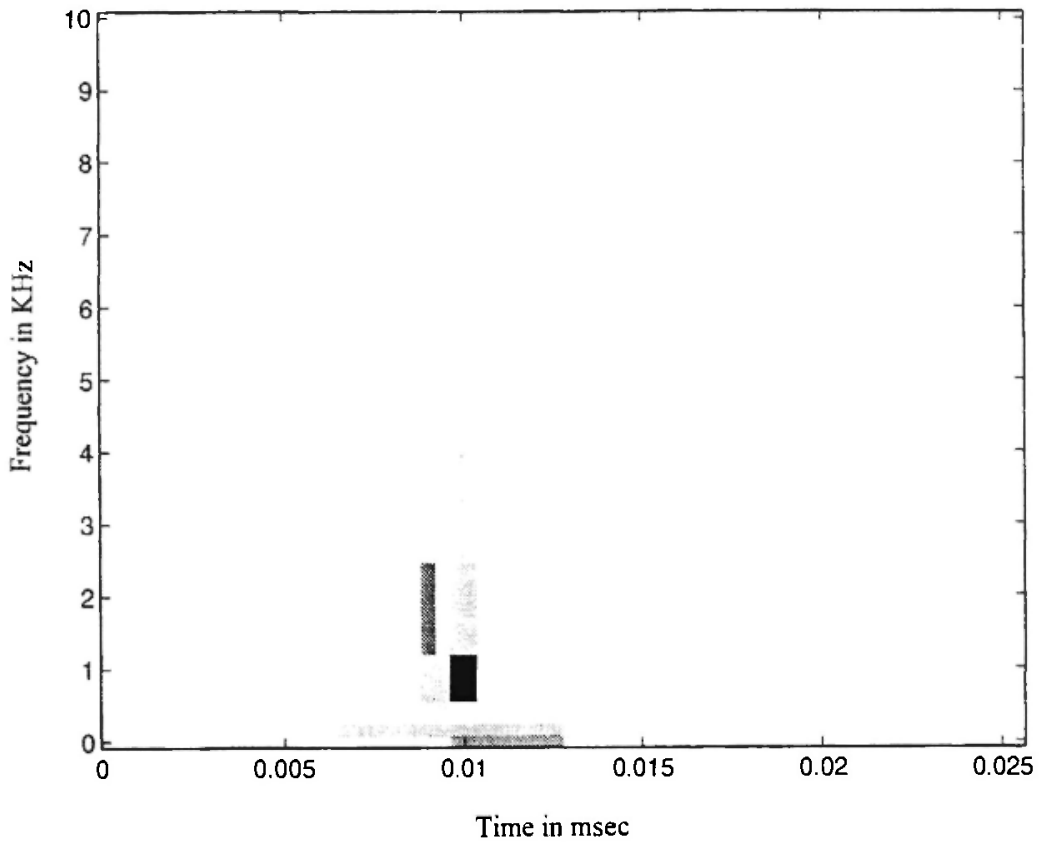

Fig. 1: An EMG study of a MYO subject. d) Average autoregressive and cepstral coefficients. e) Average time-frequency plot of wavelet coefficients DAU4 (black = maximum power, white $=$ minimum power). 
and W.F. Fincham
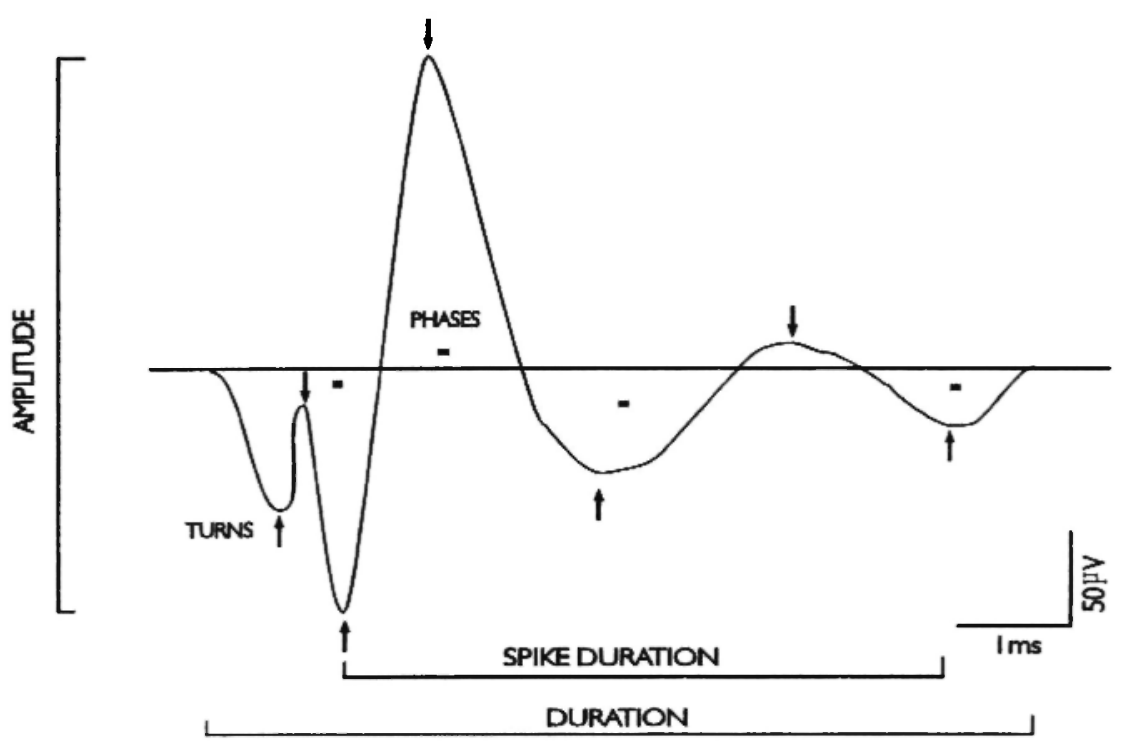

Fig. 2: MUAP time domain parameters.

area, number of phases and number of turns (see also Fig. 1b). The definition of the time domain parameters is given in appendix A.1, and their statistics for the NOR, MYO and MND groups are tabulated in table A.1. For the NOR group the mean and standard deviation values computed for duration, amplitude, and number of phases were $9.60 \pm 2.75 \mathrm{~ms}, 0.376 \pm$ $0.306 \mathrm{mV}$, and $2.6 \pm 0.8$, respectively. For the MYO group the mean and standard deviation values for duration, amplitude, and number of phases were $7.15 \pm 2.34 \mathrm{~ms}, 0.314 \pm 0.250 \mathrm{mV}$, and $2.7 \pm 1.0$, respectively. MUAPs with short duration and reduced amplitude are typical findings in patients suffering from myopathy. These findings are attributed to fiber loss within the motor unit, with the degree of reduction of these parameters reflecting the amount of fiber loss. For the MND group, the mean and standard deviation values for duration, amplitude, and number of phases were $13.42 \pm$ $3.86 \mathrm{~ms}, 0.614 \pm 0.426 \mathrm{mV}$, and $4.0 \pm 1.8$, respectively. Typically the mean durations of the MUAPs are longer and the mean amplitudes and the number of phases are increased in patients suffering from motor neuron 
disease. In this disease there is an increase in the number or density of the fibers in the motor unit due to renervation of the motor units. There is also an increase in the temporal dispersion of the activity picked up by the recording electrode. This effect is the result of slowed conduction along the terminal branches of individual nerve fibers, increase in the end-plate zone, or both.

\subsubsection{Frequency domain parameters.}

The following frequency domain parameters were computed from the MUAP waveforms as described in appendix sections A.2 and A.3: spectral moments of order 0,1 and $2\left(M_{0}, M_{1}\right.$, and $\left.M_{2}\right)$, median frequency and quality factor (see Fig. 1c). Table A.2 tabulates the statistics of the frequency domain parameters for the NOR, MYO and MND groups. For the NOR, MYO and MND group the mean and standard deviation of the median frequency were $413 \pm 285 \mathrm{~Hz}, 629 \pm 344 \mathrm{~Hz}$, and $339 \pm 241 \mathrm{~Hz}$ respectively. In the case of the MYO group the power spectrum is shifted towards higher frequencies compared to NOR, whereas in the case of the MND group the power spectrum is shifted towards lower frequencies compared to NOR. Spectral moment $M_{0}$ is actually the average power of the spectrum. Spectral moment $M_{0}$ was bigger in the MYO group than in the NOR group. $M_{0}$ in the MYO group was $29.70 \mu \mathrm{V}^{2}$ and in the NOR group was $20.71 \mu \mathrm{V}^{2}$. Conversely, $M_{0}$ in the MND group was $15.30 \mu \mathrm{V}^{2}$, being smaller than $M_{0}$ in the NOR group. Spectral moments $M_{1}$ and $M_{2}$ represent the area of the power spectrum after being weighted by frequency raised to the power of one and two respectively. Thus, since all the frequency parameters in the MYO group were found to be of higher power level than the NOR group, it follows that $M_{1}$ and $M_{2}$ in the MYO group would be greater than $M_{1}$ and $M_{2}$ in the NOR group, with the reverse being true for the MND group.

\subsubsection{Autoregressive coefficients.}

The autoregressive (AR) model order $p$ and coefficients $\alpha_{1}$ to $\alpha_{12}$ were computed as given in appendix A.2 (see Fig. Id). Table A.3 tabulates the 
and W.F. Fincham

statistics of the AR coefficients for the NOR, MYO and MND groups. The mean and standard deviation values of model order $p$ were $6.08 \pm 3.01$ for the NOR group, $5.98 \pm 3.28$ for the MYO group and $7.67 \pm 4.19$ for the MND group. These results indicated that 12 AR coefficients were adequate for modelling the MUAP signals in the three groups investigated. Also, results showed that higher model orders were needed for the MND subjects compared to the NOR subjects due to the higher complexity in shape. On the contrary, lower model orders were obtained for the MYO group compared to the NOR group due to the overall simpler MUAP waveforms in the MYO group.

\subsubsection{Cepstral coefficients.}

The cepstral coefficients were computed from the AR coefficients as described in appendix A.4 (see Fig. 1d). Table A.4 tabulates the statistics of the cepstral coefficients $c_{1}$ to $c_{12}$ for the NOR, MYO and MND groups. The motivation for using the cepstral coefficients is their high discrimination ability documented in speech recognition compared to the AR coefficients (Atal, 1976).

\subsubsection{Wavelet coefficients.}

The wavelet transform (WT) provides a linear two dimensional timefrequency representation and was investigated for describing motor unit action potential (MUAP) morphology. The WT was investigated because it has the ability to localize the changes in the statistics of nonstationary signals and it provides an alternative to the classical Short-Time Fourier Transform (STFT) which uses a single analysis window (Rioul and Vetterli, 1991). The WT uses short windows at high frequencies and long windows at low frequencies. It was shown that MUAP signals can be represented using a small number of significant WT coefficients that are located around the main spike (Pattichis et al., 1996). Also, due to the nature of the WT high frequency coefficients are highly localized in time and capture the location of MUAP spike changes whereas low frequency coefficients provide lower 
time localization describing the slow components of the signal. This is clearly illustrated in Fig. le.

Four different wavelets were computed as described in appendix A.5: Daubechies 4 (DAU4) and 20 (DAU20) with 4 and 20 coefficients respectively, Chui $(C H)$ and Battle-Lemarie $(B L)$. Analysis was carried out for six wavelet bands as follows (see also Table A.5). Scale $d^{I}$ represents the highest frequency bandwidth $5000-10000 \mathrm{~Hz}$, and the lowest time bandwidth $0.1 \mathrm{~ms}$ with 256 points. The next scale, $d^{2}$, represents the next lower frequency bandwidth, $2500-5000 \mathrm{~Hz}$, with the time bandwidth doubled, $0.2 \mathrm{~ms}$, and with half the number of data points, and so on. Note that the wavelet band $d^{\sigma}$ represents the lower bandwidth for the detail coefficients, $156.25-312.5 \mathrm{~Hz}$ with $3.2 \mathrm{~ms}$ time bandwidth and 8 points, whereas $s^{6}$ represents the coarse coefficients, covering the frequencies 0 $156.25 \mathrm{~Hz}$, with the same time bandwidth and number of points. The normalized MUAP wavelet power distribution per band per group for DAU4, DAU20, $C H$ and $B L$ was computed (see Table A.6). It is shown that more than $85 \%$ of the power is concentrated in the lower four bands $d^{-4}, d^{5}$, $d^{\sigma}$ and $s^{\sigma}$. Also, for all the wavelets for the MND group there is a shift towards lower frequency bands, whereas for the MYO group there is a shift towards higher frequency bands when compared to the NOR group (Pattichis et al., 1996). These findings are in agreement with frequency analysis as presented above in section 2.2.2. A total of 16 coefficients around the main spike were extracted, four from each of the following bands: $d^{4}$, $d^{5}, d^{6}$ and $s^{6}$. These 16 coefficients were used as the input vector for the classification.

3 Modular Neural Network Decision Support System

\subsection{Modular Neural Networks}

According to Haykin (1994), a neural network is said to be modular, if the computation performed by the network can be decomposed into modules or subsystems which operate on distinct inputs without communicating with 
and W.F. Fincham

each other. The outputs of the modules are combined in order to form the final output of the system. Modular networks allow complex learning problems to be solved by dividing the problem into a set of subproblems, each of which may be easier to solve than the original problem (Jacobs and Jordan, 1991; Jordan and Jacobs, 1995). In addition, modular networks allow, without any difficulty, the combined use of supervised and unsupervised learning paradigms or different learning algorithms. This combines the advantages of the individual modules and can improve the overall performance. Modularity also seems to be an important principle in the architecture of biological nervous systems. For example, the complex computations performed by the visual system are broken down into smaller subtasks, allowing specific modules to be specialised for specific computation tasks. Advantages of modular networks include higher learning speed, better data representation and easier hardware implementation since complex problems can be split into simpler and more manageable subproblems (Haykin, 1994).

More specifically, in the case of difficult pattern recognition problems, the combination of the outputs of multiple classifiers using for input multiple feature sets extracted from the raw data, can substantially improve the overall classification performance. In the case of noisy or of a limited amount of data, different classifiers often provide different generalisations by realising different decision boundaries. Also, different feature sets provide different representations of the input patterns, containing different classification information. Selecting the best classifier or the best feature set is not necessarily the ideal choice, since potentially valuable information contained in the less successful feature sets or classifiers may be lost. The combination of the results of the different features and the different classifiers increases the probability that the errors of the individual features or classifiers may be compensated by the correct results of the rest (Tumer and Ghosh, 1996).

In this work a modular neural network decision support system was implemented for the classification of MUAPs into a disease class. The system consists of the following two distinct subsystems. In the first 
subsystem the statistics of multiple features extracted from the individual MUAPs obtained by a subject were fed into multiple neural network classifiers and the classification results were combined. In the second subsystem multiple MUAPs obtained by a subject were fed sequentially to the system and then the classification results were combined. The outputs of the two subsystems were further combined in order to improve the diagnostic yield Figure 3 illustrates the flowchart of the modular neural network diagnostic system. The system consists basically of three components: feature extractors, classifiers, and combiners. Different realisations of these components were implemented, with detailed analysis given in the rest of the section.

\subsection{Subsystem 1: Multi-Feature, Multi-Classifier}

The combination of eight different feature sets extracted from the MUAP waveforms and three different neural network classifiers were investigated for the classification of EMG signals into a disease class (see Fig. 3). The classification results of the different feature sets and the different classifiers were combined using majority voting in order to improve the diagnostic yield.

3.2.1 Multiple features. For each subject the average vector of 20 MUAPs per subject for each feature set was computed and used as input to the classifiers (see Fig. 3). The following eight different feature sets were extracted from the MUAP waveforms as described in section 2.2 and analysed in detail in appendix $\mathbf{A}$.

(i) Time domain parameters $(n=7) \quad$ : duration, spike duration, amplitude, area, spike area, number of phases and number of turns.

(ii) Frequency domain parameters $(n=5)$ : spectral moments of order 0 , 1 and 2 , median frequency and quality factor. 


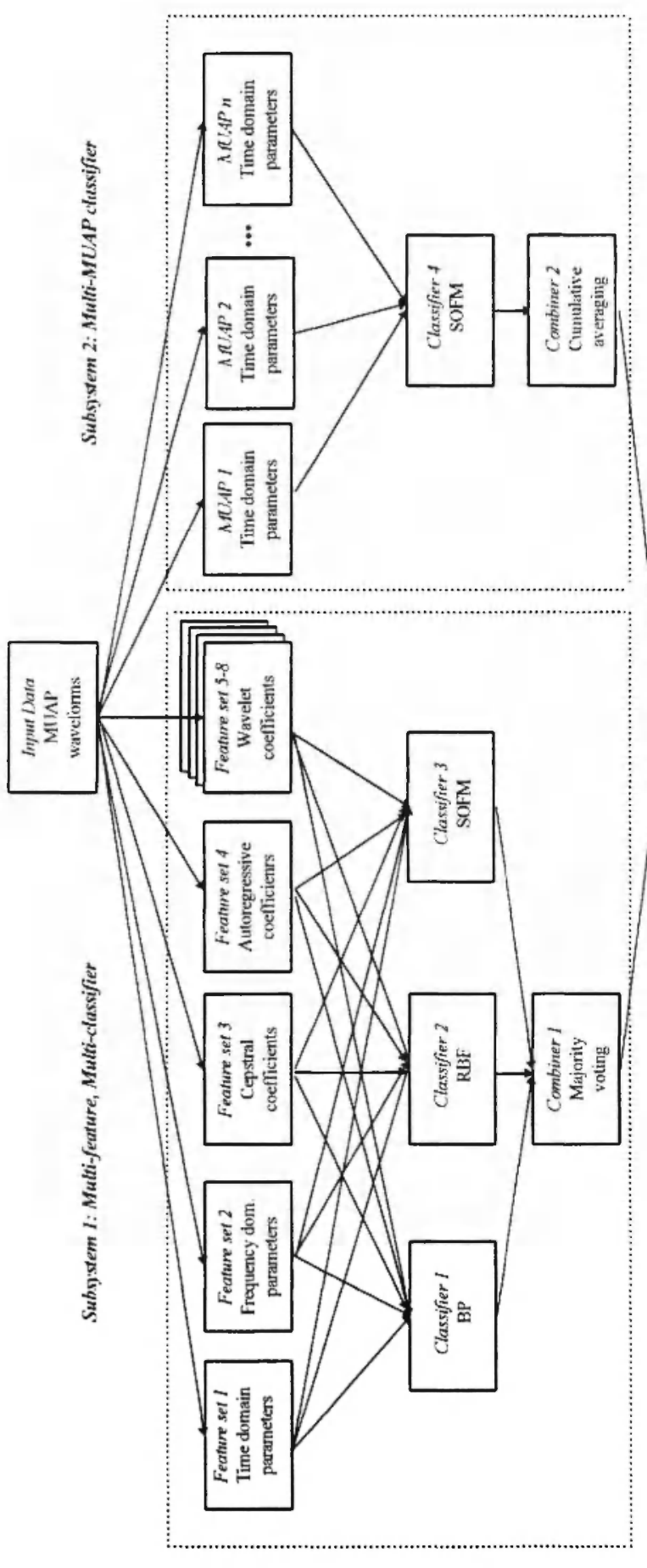

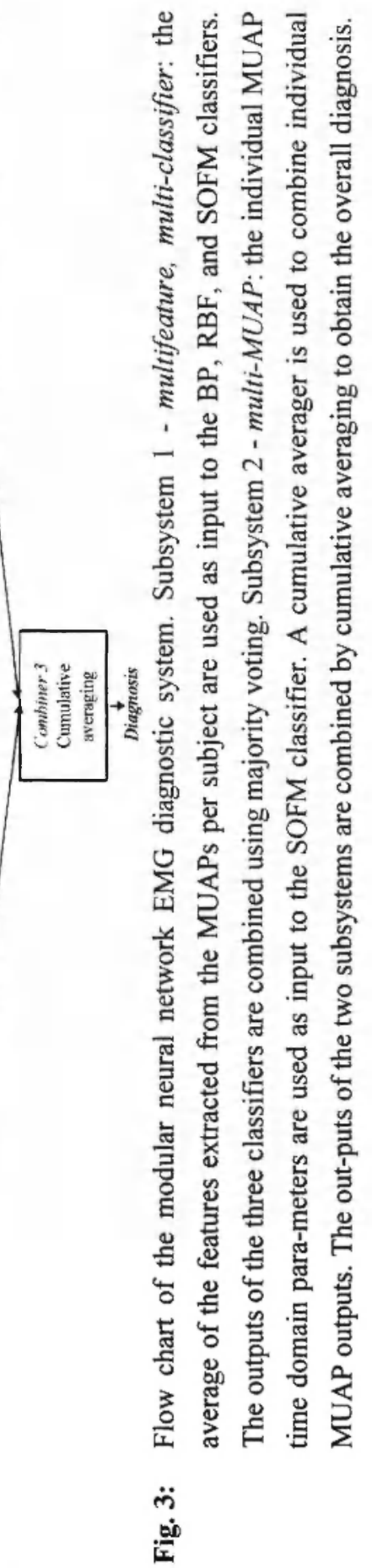

115 
(iii) Autoregressive, coefficients $(n=12) \quad$ : $\alpha_{1}$ to $\alpha_{12}$.

(iv) Cepstral coefficients $(n=12)$

$\therefore c_{1}$ to $c_{12}$.

(v) Wavelet transform DAUA $(n=16)$

$\therefore s_{2}^{-6}$ to $s_{5}^{-6}, d_{2}^{-6}$ to $d_{5}{ }^{-6}, d_{6}{ }^{5}$ to $d_{9}^{5}, d_{11}^{-4}$ to $d_{14}^{-4}$.

(vi) Wavelet transform DAU20 $(n=16)$ : $s_{2}^{-6}$ to $s_{5}^{-6}, d_{2}^{-6}$ to $d_{5}^{-6}, d_{6}{ }^{5}$ to $d_{9}{ }^{5}, d_{11}^{-4}$ to $d_{14^{-4}}$.

(vii) Wavelet transform $\mathrm{CH}(n=16)$ $\therefore s_{2}{ }^{\sigma}$ to $s_{5}{ }^{-\sigma}, d_{2}{ }^{\sigma}$ to $d_{5}{ }^{\sigma}, d_{6}{ }^{-5}$ to $d_{9}^{-5}, d_{11}^{-4}$ to $d_{14}^{-4}$.

(viii) Wavelet transform $B L(n=16)$ $\therefore s_{2}{ }^{-6}$ to $s_{5}{ }^{-6}, d_{2}{ }^{\sigma}$ to $d_{5}{ }^{-6}, d_{6}{ }^{5}$ to $d_{9}^{-5}, d_{11^{-4}}$ to $d_{14^{-4}}$.

The time domain and the frequency domain parameters were further normalised by division with their mean values.

\subsubsection{Multiple classifiers.}

In this work both supervised and unsupervised learning techniques were used for training the ANN models. Three different classifiers were implemented using the following algorithms as shown in Fig. 3.

(i) Backspropagation algorithm. The supervised learning error backpropagation (BP) algorithm (Rumelhart et al., 1986) with momentum and adaptive learning rate was used for training a three layer feedforward network. The implementation given by the MATLAB neural networks toolbox was used (Demuth and Beale, 1994). Momentum prevents the network from getting trapped at a shallow local minimum of the error surface, leading to a failure to converge and subsequently in reaching a global minimum. Momentum is added by making weight changes $\Delta w(t+1)$ equal to the sum of a fraction of the last weight change $\Delta w(t)$ and the new change derived by the BP algorithm. The BP algorithm with momentum is expressed mathematically as follows:

$$
\Delta w(t+1)=m \Delta w(t)+(1-m) x \eta \delta
$$


and W.F. Fincham

where $m$ is the momentum constant set typically to $0.95, x$ are the input values, $\eta$ the learning rate and $\delta$ is the error signal given by the BP algorithm. Also, in order to speed up learning, an adaptive learning rate $\eta$ can be used. If the new error exceeds the old error by more than a predefined ratio, no weight change takes place and the learning rate decreases. If the error is less, then the learning rate is increased.

(ii) Radial-basis function network. The radial-basis function (RBF) network was used for supervised learning (Broomhead and Lowe, 1986; Chen et al., 1991). The network was trained using an incremental solver, adding iteratively one neuron per epoch until a preset sum squared error goal is met or the maximum number of neurons is reached. The RBF network implemented by the MATLAB neural networks toolbox was used (Demuth and Beale, 1994). The output of each neuron is given by

$$
y=R B F(\|x-w\| 0.8326 / S p)
$$

where $R B F$ is a Gaussian function, $\|x-w\|$ is the Euclidean distance between the input vector $x$ and the weight vector $w$ and $S p$ is the spread parameter. The output increases as the distance between the input and weight vectors decreases. The spread parameter should be large enough so that the RBF neurons respond to overlapping regions of the input space but not so large that all the neurons respond in essentially the same manner.

(iii) Self-Organising feature map algorithm. The self-organising feature map (SOFM) algorithm is an unsupervised learning algorithm where the input patterns are freely distributed over the output node matrix (Kohonen, 1990, 1995). The weights are adapted without supervision in such a way, so that the density distribution of the input data is preserved and represented on the output nodes. This mapping of similar input patterns to output nodes which are close to each other represents a discretisation of the input space, allowing a visualization of the 
distribution of the input data. The output nodes are usually ordered in a two dimensional grid, and the weights of the output nodes in the neighborhood of the winning output node are adapted simultaneously. The neighborhood around the winning output node, decreases in size over time. The weights of the output nodes in the neighborhood are adapted with

$$
w(t+1)=w(t)+\eta h(t)(x-w(t))
$$

where $\eta$ is the learning rate $(0<\eta \leq 1)$ and $h(t)$ is a decreasing function which decreases with time.

At the end of the training phase, the output nodes were labelled with the class of the majority of the input patterns of the training set, assigned to each node. In the evaluation phase, an input pattern was assigned to the output node with the weight vector closest to the input vector, and it was said to belong to the class label of the winning output node where it had been assigned.

\subsubsection{Classification results combiner.}

The classification results of the three classifiers were combined in three different ways, using simple majority voting in order to improve the diagnostic yield. The classification results combiner is shown in Fig. 3 as combiner 1 .

(i) Combining the outputs for the eight different feature sets for each classifier. The eight different feature sets listed in section 3.2.1 were applied to the BP, RBF and SOFM classifiers. Each feature set applied to a classifier yielded an output, indicating whether the specific subject belongs to the NOR, or MYO or MND group. The eight outputs corresponding to the eight feature sets of each classifier were combined using majority voting. The subject was assigned to the class with the maximum votes. 
and W.F. Fincham

(ii) Combining the results of the three classifiers. As described above, the combination of the eight feature sets by each classifier yielded an output result. The three output results of the three classifiers were further combined using majority voting.

(iii) Combining the outputs derived from the eight different feature sets from the three classifiers. The 24 output results of the eight feature sets applied to the three classifiers were combined using majority voting. The highest diagnostic yield was obtained by the combination of the 24 classification results of all feature sets from all classifiers.

\subsection{Subsystem 2: Multi-MUAP Classifier}

In the multi-feature, multi-classifier diagnostic subsystem the input feature set vectors consisted of the average of the features extracted from the 20 MUAPs describing each subject. Another way to structure the input data was to evaluate each MUAP waveform or feature set separately and then to combine the classification results for the 20 MUAPs for each subject. The latter procedure has the advantage that valuable information contained in the individual MUAPs may not be lost during the averaging process. The multi-MUAP classifier subsystem is shown in Fig. 3.

\subsubsection{Multi-MUAP features.}

Two different feature sets were used as input to the subsystem:

(i) Time domain parameters. The following time domain parameters were extracted from each MUAP waveform: duration, spike duration, amplitude, area, spike area, number of phases and number of turns. Thus, for each subject a vector of $20 \times 7$ was computed. The time domain parameters were chosen because they have the advantage that no transformation is needed, and due to their better representation capability compared to the other features as described in the following section.

(ii) MUAP waveform vectors. The original MUAP matrix of $20 \times 512$ samples was downsampled by two in order to reduce its dimensionality. 
The newly obtained 20x256 samples MUAP matrix was further reduced to $20 \times 60$ samples by extracting from each MUAP waveform 60 samples $(6 \mathrm{~ms})$ containing only the main spike of the MUAP waveform.

\subsubsection{SOFM classifier.}

The SOFM is an unsupervised learning classifier where the input patterns are freely distributed over the output node matrix with no specific class labels assigned. This allows the use of the individual MUAP waveforms or their parameters as input regardless of the overlap among the disease classes. The SOFM network was trained as described in section 3.2.2. In this subsystem, the feature set vector for each $M$ : AP was fed into the SOFM algorithm sequentially. Thus, during the training phase, the 480 MUAPs of the training set (20 MUAPs $\times 24$ subjects) were distributed over a $15 \times 15$ output node matrix (see Fig. 4 in section 4). MUAPs with similar features were assigned to neighbouring nodes.

\subsubsection{Multi-MUAP combiner.}

The classification results of the individual MUAPs were combined using cumulative averaging. The multi-MUAP combiner is shown in Fig. 3 as combiner 2. At the end of the training phase, each output node was labelled based on the number of MUAPs per class assigned to each winning output node. For example, in an output node 2, 6, and 2 MUAPs were assigned belonging to classes NOR, MYO and MND respectively. This output node was labelled with the following percentage scores: NOR $20 \%$, MYO $60 \%$ and MND 20\%. During the evaluation phase, the 20 MUAPs per subject were fed sequentially into the classifier and each MUAP was assigned to a winning output node. The percentage scores of the winning output nodes were accumulated and averaged, and the subject was classified to the disease class with the greatest score. 
and W.Fi Fincham

\subsection{System Combiner}

The outputs of the two subsystems were further combined using cumulative averaging in order to obtain the overall diagnostic yield, see combiner 3 in Fig. 3. For this purpose the percentages of the classification results, of the 24 outputs of all feature sets from all classifiers of the multifeature, multi-classifier subsystem for each subject, and the output of the multi-MUAP classifier subsystem were accumulated and averaged. The subject was assigned to the class with the highest score.

\section{Results}

A total of 480 MUAPs obtained from 24 subjects, 8 NOR, 8 MYO and 8 $\mathrm{MND}$, were used for training the ANN classifiers, whereas a total of 320 MUAPs, obtained from 16 subjects, 4 NOR, 5 MYO and 7 MND were used for evaluation. Due to the rather limited amount of the input data and in order to verify the correctness of the classification results a bootstrapping procedure was used. The system was trained and evaluated using five different bootstrap sets where in each set 24 different subjects were selected at random for training and 16 different subjects for evaluation. The mean percentage and the standard deviation (Std) of the correct classifications score, i.e. diagnostic yield, for the five bootstrap sets was computed for each classifier, for the eight different feature sets. Table 1 tabulates the mean and Std of the diagnostic yield of the modular neural network EMG diagnostic system for the five sets. For the multi-feature, multi-classifier analysis the classification results are given for each feature set per classifier and the results by the combination of features and clāssifiers. For the multi-MUAP analysis the classification results are given for the time domain parameters and the MUAP waveforms. Furthermore the overall diagnostic yield is given for the whole system.

For the multi-feature, multi-classifier subsystem, a total of 120 runs (8 feature sets $\times 3$ classifiers $\times 5$ bootstrap sets) were carried out. For each feature set the average diagnostic yield for the BP, RBF, and SOFM 
Table 1

Mean and SD of the diagnostic yield (DY\%) for the evaluation of the modular neural network EMG diagnostic system, illustrated in Fig. 3 after bootstrapping the available data for five different sets of subjects.

\begin{tabular}{|c|c|c|c|c|c|c|}
\hline Feature set & $n$ & $\begin{array}{c}B P \\
D Y \%\end{array}$ & $\begin{array}{l}R B F \\
D Y \%\end{array}$ & $\begin{array}{c}S O F M \\
D Y \%\end{array}$ & $\begin{array}{c}\text { Average } \\
\text { DY\% }\end{array}$ & $\begin{array}{c}\text { Combiners } \\
D Y \%\end{array}$ \\
\hline \multicolumn{7}{|c|}{ Subsystem I: Multi-Feature, Multi-Classifier } \\
\hline Time domain & 7 & $81.2 \pm 11.8$ & $72.5 \pm 8.5$ & $812 \pm 5.5$ & $78.3 \pm 8.6$ & \\
\hline Frequency domain & 5 & $63.7 \pm 2.5$ & $60.0 \pm 6.3$ & $63.7 \pm 10.1$ & $62.5 \pm 6.3$ & \\
\hline Autoregressive & 12 & $56.2 \pm 7.9$ & $50.0 \pm 6.8$ & $47.5 \pm 10.1$ & $51.2 \pm 8.3$ & \\
\hline Cepstral & 12 & $62.5 \pm 15.8$ & $60.0 \pm 5.0$ & $68.7 \pm 3.9$ & $63.7 \pm 8.2$ & \\
\hline Wavelet $D A U 4$ & 16 & $68.7 \pm 5.5$ & $60.0 \pm 5.0$ & $70.0 \pm 6.1$ & $66.2 \pm 5.5$ & \\
\hline Wavelet $D A U 20$ & 16 & $57.5 \pm 8.3$ & $55.0 \pm 4.6$ & $66.2 \pm 6.3$ & $59.6 \pm 6.4$ & \\
\hline Wavelet $\mathrm{CH}$ & 16 & $65.0 \pm 6.3$ & $56.2 \pm 11.8$ & $68.7 \pm 3.9$ & $63.3 \pm 7.3$ & \\
\hline Wavelet $B L$ & 16 & $65.0 \pm 101$ & $66.2 \pm 8.5$ & $66.2 \pm 5.0$ & $65.8 \pm 7.9$ & \\
\hline Average & 16 & $65.0 \pm 8.5$ & $60.0 \pm 7.1$ & $66.5 \pm 6.4$ & $63.8 \pm \overline{7} . \overline{3}$ & \\
\hline Combine features ${ }^{2}$ & & $73.7 \pm 7.2$ & $72.5 \pm 5.0$ & $78.7 \pm 6.3$ & $75.0 \pm 6.2$ & \\
\hline \multicolumn{6}{|l|}{ Combine classifiers ${ }^{\top}$} & $80.0 \pm 6.1$ \\
\hline \multicolumn{6}{|c|}{ Combine all features from all classifiers ${ }^{+}$} & $82.5 \pm 4.6$ \\
\hline
\end{tabular}

\begin{tabular}{|l|l|l|l|l|c|}
\hline \multicolumn{4}{|l|}{ Subsystem 2: Multi-MUAP Classifier } \\
\hline Time domain & $20 \times 7$ & & $87.5 \pm 7.9$ & & $87.5 \pm 7.9$ \\
\hline MUAP waveforms & $20 \times 60$ & & $72.5 \pm 5.0$ & & $72.5 \pm 5.0$ \\
\hline
\end{tabular}

\section{Combine: Subsytems 1 and 2}

Overall diagnosis

\footnotetext{
'Feature set vector size

2.1.4 Sec section 3.2 .3 , (i), (ii), and (iii) respectively
}

In subsystem 1, multi-feature, multi-classifier, the average feature vector for each subject for eight different feature sets were fed to three different classifiers, and their results were combined. In subsystem 2, multi-MUAP classifier, the individual MUAP time domain parameters and MUAP waveforms were used as input to the SOFM classifier. The outputs of the two subsystems were combined to obtain the overall diagnosis. 
and W.F. Fincham

classifiers were computed. The best feature set was the time domain parameters with average diagnostic yield $78.3 \%$, followed by the wavelet transform coefficients $D A U 4$ with $66.2 \%$, and the $B L$ with $65.8 \%$. A similar average diagnostic yield, in the region of $63 \%$ was obtained by the cepstral coefficients, the wavelet transform coefficients $\mathrm{CH}$, and the frequency domain features. The wavelet transform coefficients DAU20 yielded $59.6 \%$, and the autoregressive coefficients yielded the lowest diagnostic yield which was $51.2 \%$. For each classifier, the average diagnostic yield was computed for all eight feature sets. The best classifier in average was the SOFM with $66.5 \%$, followed closely by the BP classifier with $65.0 \%$ and the RBF classifier with $60.0 \%$. The combination of the classification results of the multiple features per classifier, enhanced the diagnostic yield and exceeded significantly the average results achieved by each classifier. For the SOFM classifier, the diagnostic yield by the combination of all feature sets was $78.7 \%$ compared to $66.5 \%$ for the average yield, for the BP classifier $73.7 \%$ compared to $65.0 \%$, and for the RBF classifier $72.5 \%$ compared to $60.0 \%$. The combination of the combined results of the three classifiers improved the diagnostic yield further to $80.0 \%$. The best diagnostic yield was achieved by the combination of the 24 classification results of all feature sets from all classifiers and it was $\mathbf{8 2 . 5 \%}$. This diagnostic yield was higher than the diagnostic yield of the best feature set from the best classifiers, which was $81.2 \%$ for the time domain parameters and the SOFM and BP classifiers.

For the multi-MUAP classifier subsystem, the classification performance was enchanced with the diagnostic yield being $87.5 \%$, when the time domain parameters were used as input into the SOFM classifier. The use of the MUAP waveform vectors as input, performed poorer and yielded $72.5 \%$. This suggests that the time domain parameters result into a better generalisation due to their lower dimensionality and probably due to the more general information they carry.

The outputs of the two subsystems were further combined using cumulative averaging in order to obtain the overall diagnostic yield. For this purpose the combinations with the best outputs of the two subsystems were used. These were the combination of the 24 outputs of all feature sets from 
all classifiers of the multi-feature, multi-classifier subsystem, and the output of the multi-MUAP classifier subsystem using the time domain parameters. In average, the overall diagnostic yield was $87.5 \%$.

Figure 4 illustrates how the time domain parameters of the 160 MUAPs from each one of the three different classes of the training set are distributed over the output matrix of the SOFM network. It is clearly illustrated that there is a high degree of overlap among the three classes.

The ANN classifiers were implemented using the BP, the RBF and the SOFM algorithms as they were given in the MATLAB neural networks toolbox (Demuth and Beale, 1994). For the BP several network architectures were tested. In general, an architecture which gave good results for the BP algorithm was the one with 40 neurons in the first hidden layer and 20 neurons in the second hidden layer. The RBF algorithm required 22 neurons for learning the input patterns at a sum squared error goal set to 0.02 . The spread parameter $S p$ was varied depending on the feature set in order to achieve the best possible performance. For the SOFM algorithm a $5 \times 5$ output node matrix was used in the case of the multi-feature, multi-classifier subsystem, and a $15 \times 15$ output node matrix in the case of the multi-MUAP subsystem. The $\overline{B P}$ classifier was trained for 5000 learning epochs whereas the $\mathrm{RBF}$ algorithm converged very fast requiring only 22 learning epochs. The SOFM algorithm was trained for 1000 learning epochs and in general it was faster than the BP algorithm and slower than the RBF algorithm. In every learning epoch all the 24 subjects of the training set were presented to the networks.

\section{Discussion}

This study shows that modular neural networks can be used successfully in EMG decision making in mimicking the tasks carried out by the expert neurophysiologist in MUAP analysis: (i) in comparing the statistics of MUAP features to normal reference values, and (ii) in the sequential visual assessment of MUAP waveforms. The system is completely automated, minimising observer 
and W.Fi Fincham
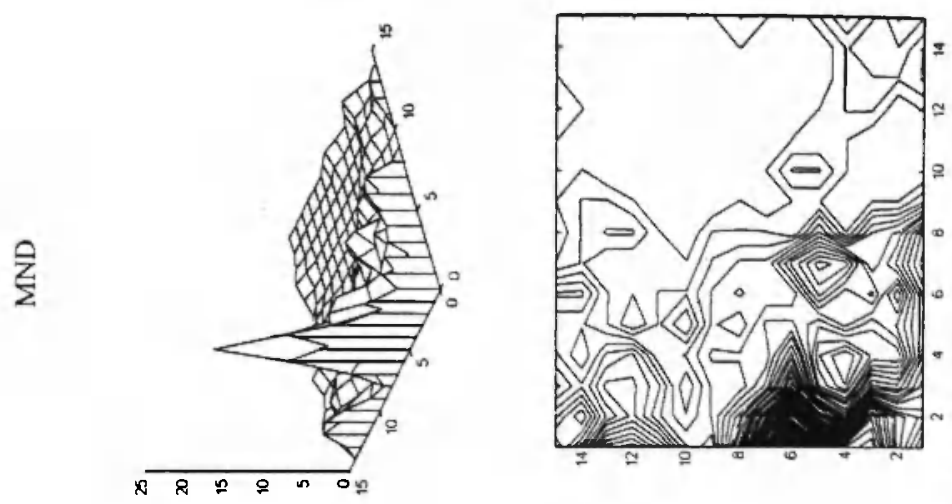

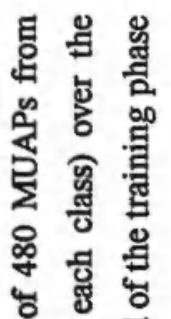
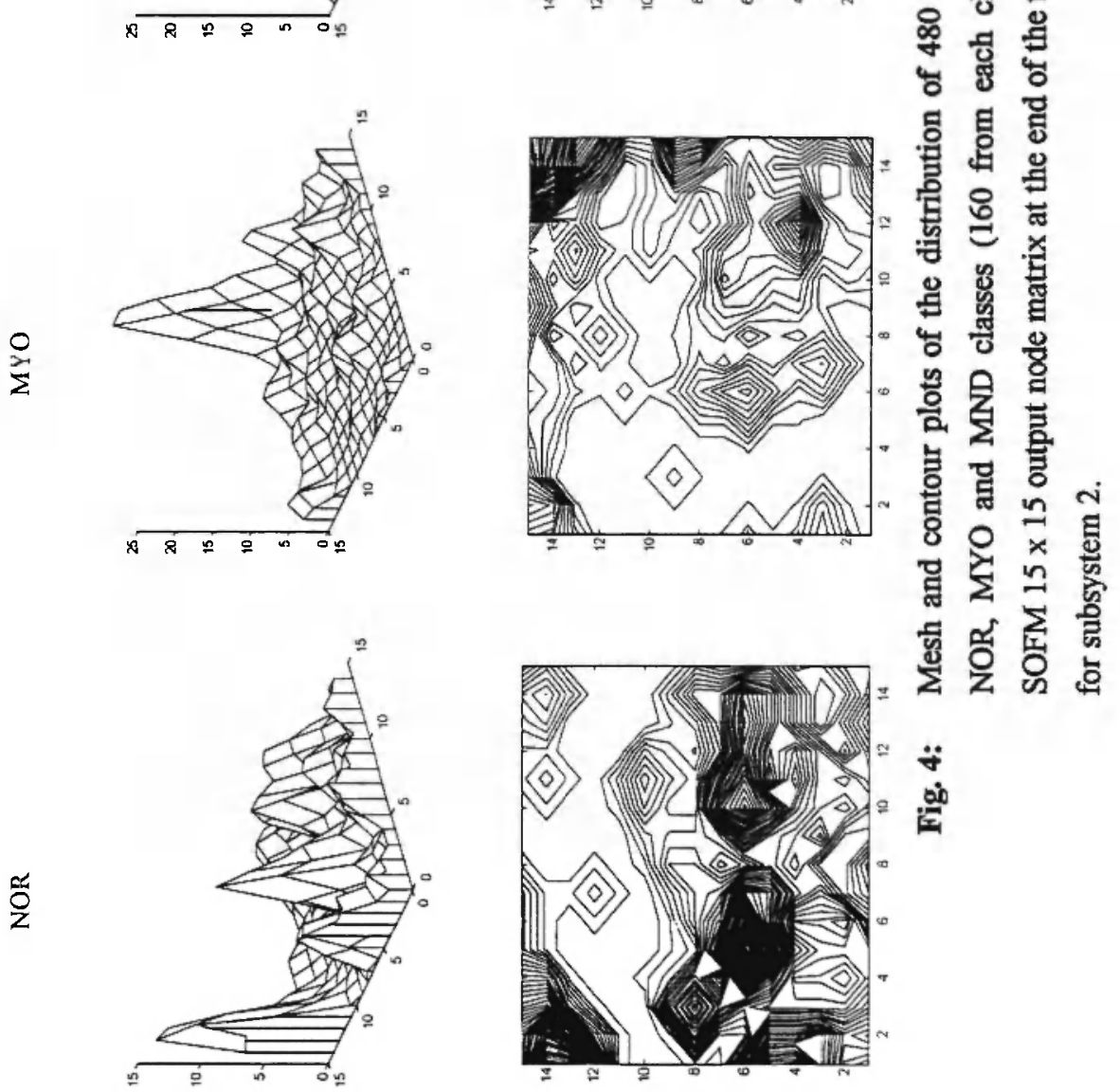
bias, facilitating the comparison of results across neurophysiologists and different methodologies, and more importantly in providing useful information for assisting the physician in reaching a more accurate diagnosis.

In this paper we have presented how the combination of different features and different neural network classifiers can be used in order to improve the classification performance of individual features or classifiers. This results a diagnostic system which combines different features sets and different classifiers to be more robust and reliable. In the multi-feature multi-classifier subsystem, the ranking of the average performance of the different feature sets is as follows: time domain parameters, wavelet coefficients $D A U 4$ and $B L$, cepstral coefficients, wavelet coefficients $\mathrm{CH}$, frequency domain parameters, wavelet coefficients $D A U 20$, and autoregressive coefficients. The time domain parameters gave the highest diagnostic yield. The better results obtained by the time domain parameters can be justified by the relative simple shape of the EMG signal at low to moderate force levels. At these forc levels the individual MUAPs have a distinct waveform shape which can be identified and the time domain parameters like duration, amplitude, number of phases etc. can be measured. These parameters captured most of the information characterising the MUAP waveform. From the four different wavelets investigated, DAU4 gave the highest average diagnostic yield, after the time domain parameters. The $D A U 4$ captured the information contained in the main part of the MUAP spike. The average diagnostic yield of the cepstral coefficients was better than the frequency domain parameters and the autoregressive coefficients. It is noted that even feature sets with poor classification results may contain potentially valuable information.

In the multi-MUAP classifier subsystem, the use of the features of the individual MUAPs as input to the classifier, instead of the average features characterising a subject, and the combination of the classification results of the individual MUAPs further enhanced the diagnostic yield. Two feature sets were used in this subsystem: the time domain parameters, and the MUAP waveforms. The highest diagnostic yield was obtained with the time domain parameters which was $\mathbf{8 7 . 5 \%}$. The methodology which is currently practiced is to compute the average of the time domain parameters of 20 
and W.F. Fincham

MUAPs and compare these with reference values (Buchthal, 1957; Stalberg et al., 1986; Stalberg et al., 1994). With this technique it is hoped that the characteristics of the 'abnormal' MUAPs will dominate over the characteristics of the normal ones. The results in this work proved that a higher diagnostic yield was obtained when processing each individual MUAP separately and then combining the classification results. This is due to the fact that useful distinctive information may be lost when using the statistics of the MUAP parameters per subject. The individual processing of each MUAP is much more alike to the manual process used by the physician where the individual MUAPs are visually examined in sequence, until a sufficient number of 'abnormal' MUAPs is identified in order to make a correct diagnosis. Because of the great overlap among disease classes of the individual MUAP waveforms or their parameters, the unsupervised learning SOFM algorithm was used which resulted in an efficient mapping of the MUAP data. In addition, the SOFM algorithm allows a visualisation of the distribution of the input data as shown in Fig. 4, illustrating the high degree of overlap among the three disease classes. The multi-MUAP subsystem can be further expanded by using any of the other feature sets and combining the classification results.

The overall diagnostic yield of the modular neural network system is higher than neural network models trained with the same data set (Pattichis 1992; Pattichis et al., 1995). Although the overall diagnostic yield of the system is similar to the diagnostic yield of the multi-MUAP classifier subsystem, the overall diagnostic yield score is more reliable since its computation is based on different representations of the MUAP data and different generalisations of the neural network classifiers. It is noted that a bootstrapping procedure was used in order to obtain a more reliable estimate of the diagnostic yield.

In the modular neural network decision support system presented in this work, both supervised (BP and RBF) and unsupervised (SOFM) learning algorithms were used in order to combine their properties and the different generalisations they provide. The RBF is a fast learning algorithm by employing locally tuned neurons which respond selectively to specific 
regions of the input space. The BP is much slower but provides a better generalisation in regions of the input space where little or no training data are available (Haykin, 1994). The unsupervised learning SOFM algorithm discovers similar patterns in the input data without the help of an external teacher, leading to a discretisation of the input space. Thereafter new inputs are classified according to their response to a specific cluster of the input space. As shown in this study, the use of the SOFM algorithm can give better results in cases where there is a high degree of overlap among the classes of the input data.

In this study, the strategy followed for the combination of the neural network outputs was either simple majority voting or cumulative averaging of the outputs of the different modules. This linear combination strategy may be expanded by the use of a gating network (Jacobs and Jordan, 1991), where the best feature sets and the more successful classifiers will be given a greater weight in the combination of the outputs. The gating network will be trained simultaneously with the other ANN classifiers and will serve as mediator among the classifiers. The gating network will decide based on the input pattern presented which features or classifiers should be given the greater weight in order to achieve the best performance.

\section{Conclusion}

The results in this work show that modular neural networks are a promising tool in solving difficult pattern recognition problems. The combination of different features and different classifiers improved the overall classification performance of the system. Different features provide different representations of the input data and different classifiers provide different generalisations by realising different decision boundaries. The combination of the classification results of the individual input patterns utilises all the available information which may be lost when using the statistics of the input data, and enhanced further the classification performance of the system. The diagnostic results could be verified further using more data from more subjects, both for training and 
and W.F. Fincham

evaluating the system. In addition, other forms of data like muscle biopsy, biochemical and molecular genetic findings, and clinical data may be combined into a hybrid diagnostic system for neuromuscular diseases. Finally, the proposed methodology, followed in our work can be applied for the development of modular neural network decision support systems in biosignal analysis in other domains in medicine. 


\section{APPENDIX A: ANALYSIS OF EMG FEATURE EXTRACTION}

\section{A.1 Time Domain Parameters}

The following time domain parameters were computed from the MUAP waveforms as shown in Fig. 2 (Pattichis, 1992):

(i) Duration is the time interval between the MUAP beginning and ending points which are identified by sliding a measuring window of length $3 \mathrm{~ms}$ and width $\pm 10 \mu \mathrm{V}$.

(ii) Spike duration is the time interval from the first to the last positive peak.

(iii) Amplitude is the amplitude difference between maximum negative and minimum positive peaks.

(iv) Area is the rectified MUAP integrated over the calculated duration.

(v) Spike area is the rectified MUAP integrated over the calculated spike duration.

(vi) Phases is the number of baseline crossings within duration that exceed $\pm 25 \mu \mathrm{V}$, plus one.

(vii) Turns is the number of positive and negative peaks where the difference from the preceding and following peak exceed $25 \mu \mathrm{V}$.

Table A.1 tabulates the statistics of the time domain parameters for the NOR, MYO and MND groups.

\section{A.2 Autoregressive Analysis}

The autoregressive model of the current sample of the signal $x(n)$ is described as a linear combination of previous samples plus an error term $e(n)$ which is independent of past samples:

$$
x(n)=-\sum_{k=1}^{p} a_{\mathrm{k}} x(n-k)+e(n) \quad n=0,1, \ldots, N-1
$$

where

$x(n)$ samples of the modeled signal,

$\alpha_{k} \quad$ AR coefficients, 


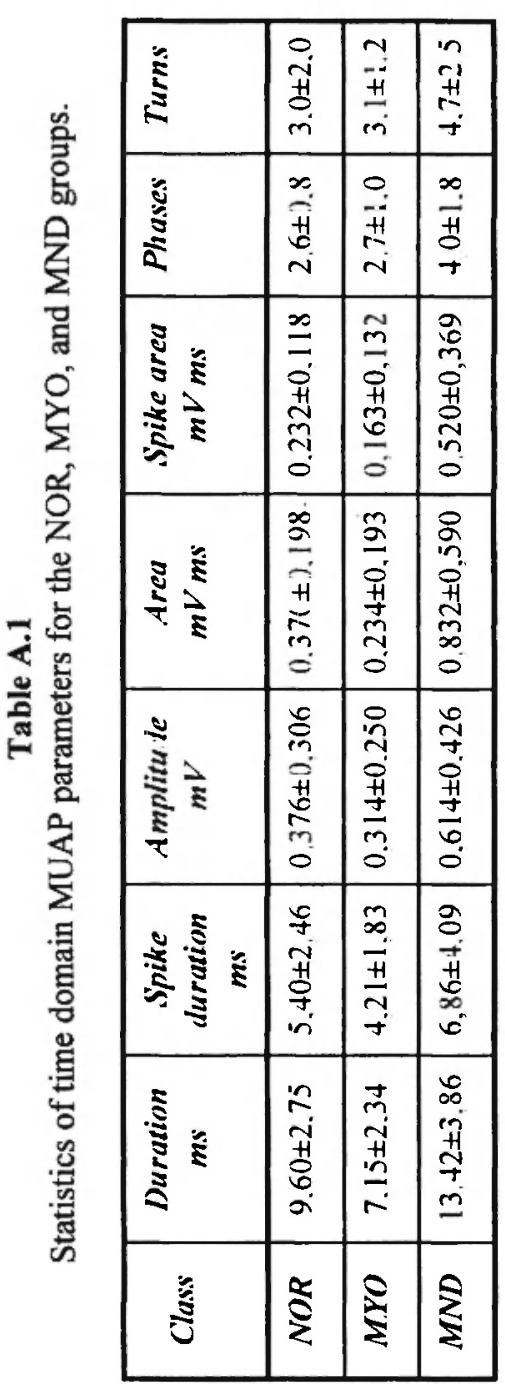

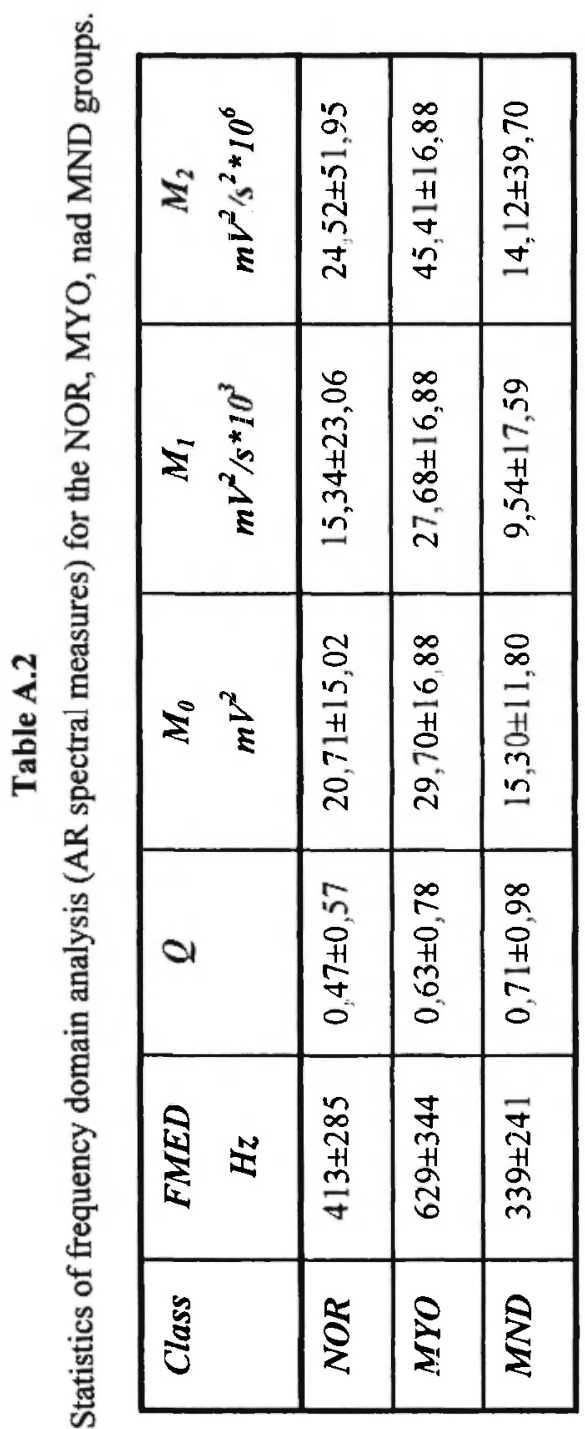


$p$ model's order,

$e(n)$ time series of residual values (prediction errors), and

$N$ is 256 samples, after downsampling by two the original MUAP series.

The model can be interpreted as a linear system with $e(n)$ as its input (white noise) and $x(n)$ its output (MUAP time series). The transfer function $H(z)$ for the AR process is:

$$
H(z)=\frac{X(z)}{E(z)}=\frac{1}{1+\sum_{k=1}^{p} \alpha_{k} z^{-k}} .
$$

The spectrum of the model can be estimated from Eq. A.2:

$$
P_{A R}(\omega)=\frac{1}{\left|1+\sum_{k=1}^{p} \alpha_{k} e^{j a k}\right|^{2}}
$$

It was assumed that the spectrum of $e(n)$ satisfies $|E(w)|=1$, i.e., for the appropriate model order $p$, it approached a white noise sequence. The AR coefficients $\alpha_{k}$ were calculated using the modified covariance algorithm as given in Marple (1987). The Akaike information criterion (AIC) (Akaike, 1974) was used for estimating the optimum AR model order:

$$
\operatorname{AIC}(p)=N \ln \left(\hat{\rho}_{p}\right)+2 p
$$

where $\hat{\rho}_{p}$ is the estimated linear prediction error variance for the model with order $p$, and $N=256$. After a model order was selected, the model fitness was checked using the residuals $e(n)$ periodogram, the normalized residual energy periodogram, the normalized cumulative residuals periodogram and the Portmanteau test statistic as described by Box and Jenkins (1976), (Elia, 1994). Table A.3 tabulates the statistics of model order $p$ and AR coefficients $;$ to $i 2$ for the NOR, MYO and MND groups. 


\begin{tabular}{|c|c|c|c|c|c|c|c|c|}
\hline \multirow{10}{*}{ 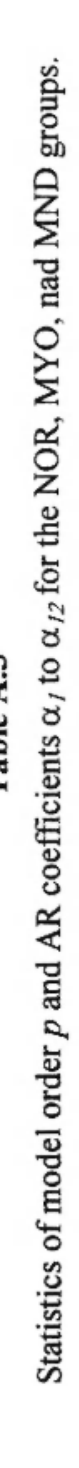 } & $\theta$ & $\begin{array}{l}\text { ह! } \\
\text { I! }\end{array}$ & $\begin{array}{l}\text { In } \\
\text { 茾 }\end{array}$ & 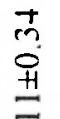 & & & & \\
\hline & & $0^{\circ}$ & $0^{\circ}$ & $0^{\circ}$ & & & & 10 \\
\hline & $\ddot{\sigma}$ & $\stackrel{n}{m}$ & $\stackrel{r}{\stackrel{n}{+}}$ & $\begin{array}{l}\text { Y } \\
\text { O } \\
\text { Hِ }\end{array}$ & $\tilde{\sigma}$ & 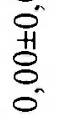 & $\begin{array}{l}O^{\prime \prime} \\
0 \\
0\end{array}$ & $\begin{array}{l}0^{\circ} \\
\text { ㅂㅇㅇ } \\
0 \\
0\end{array}$ \\
\hline & & i & 0 & P & & $a$ & 0 & $a$ \\
\hline & $\sigma^{*}$ & $\frac{\infty}{\stackrel{+}{+}}$ & 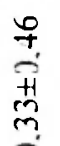 & $\begin{array}{l}\text { ก̃ } \\
\text { 草 } \\
\text { 草 }\end{array}$ & $\bar{\sigma}$ & $\begin{array}{l}0 . \\
\text { ○. } \\
\text { +1 } \\
\text { ᄋ. } \\
0 .\end{array}$ & $\begin{array}{l}\overrightarrow{0} \\
\stackrel{+}{H} \\
0 \\
0 \\
\end{array}$ & 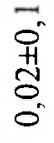 \\
\hline & $\tilde{\sigma}$ & 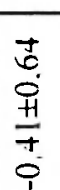 & $\begin{array}{l}\text { rn } \\
\text { on } \\
\text { H } \\
\text { bo } \\
\text { ? }\end{array}$ & 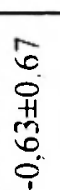 & $\bar{\sigma}$ & $\frac{\infty}{0}$ & $\begin{array}{l}\text { I } \\
\text { O } \\
\text { H1 } \\
0 \\
0 \\
0\end{array}$ & 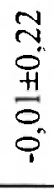 \\
\hline & $\hat{\theta}$ & 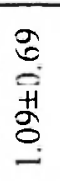 & 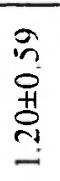 & $\begin{array}{l}N \\
\stackrel{N}{O} \\
\frac{H}{N}\end{array}$ & $\dot{\theta}$ & $\begin{array}{l}\underset{0}{\infty} \\
\stackrel{+}{+} \\
\stackrel{+}{0} \\
0 .\end{array}$ & 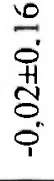 & 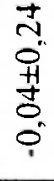 \\
\hline & $\bar{\sigma}$ & 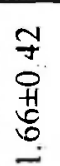 & 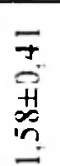 & 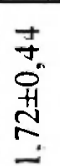 & $\theta^{\infty}$ & 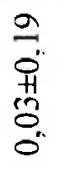 & 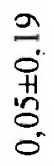 & $\begin{array}{l}0 \\
\text { d } \\
\text { o } \\
+ \\
\infty \\
0 \\
0\end{array}$ \\
\hline & $\approx$ & $\begin{array}{l}\overrightarrow{0} \\
\ddot{1} \\
0 \\
0 \\
0 \\
0\end{array}$ & 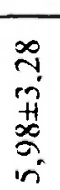 & $\begin{array}{l}a \\
\pm \\
+1 \\
0 \\
0 \\
0\end{array}$ & $\hat{\sigma}$ & 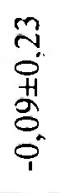 & \begin{tabular}{l}
\multirow{2}{*}{} \\
0 \\
+1 \\
0 \\
0 \\
0 \\
0
\end{tabular} & $\begin{array}{l}0 \\
\text { r. } \\
0 \\
0 \\
0 \\
0 \\
0\end{array}$ \\
\hline & $\frac{8}{0}$ & $\underset{\Sigma}{\Sigma}$ & $\sum$ & $\sum$ & $\stackrel{3}{0}$ & $\frac{5}{2}$ & $\stackrel{2}{z}$ & $\underset{\Sigma}{\xi}$ \\
\hline
\end{tabular}




\section{A.3 Spectral Estimation from the AR Model}

The following frequency and power measures were derived from the AR power spectrum of each MUAP. From the AR coefficients $\alpha_{k}$, the spectrum was estimated as given by Eq. A.3. The AR coefficients were zero padded from $\mathrm{p}+\mathrm{l}$ to $4 \mathrm{~N}$ (1024 samples), and the AR power spectrum estimate of each MUAP was normalized with its maximum power value. The following parameters were computed:

(i) Quality factor $(Q)$ is the ratio of the dominant peak frequency $F_{0}$ divided by the bandwidth $(B W)$ and is expressed as:

$$
Q=\frac{F_{0}}{B W}
$$

where the bandwidth $(B W)$ is the difference of frequencies at the upper $\left(\mathrm{F}_{2}\right)$ and lower $\left(\mathrm{F}_{1}\right)-3 \mathrm{~dB}$ points of the power spectrum and is given as:

$$
B W_{1}=F_{2}-F_{l}
$$

(ii) Moments of order 0,1 and 2: A moment $M_{j}$ of order $j$ is defined as (Lindstrom and Petersen, 1983):

$$
M_{j}=\frac{2}{(2 \pi)^{j+1}} \sum_{n=0}^{N-1} f(n)^{j} P_{A R}(f(n))
$$

Moments $M_{0} M_{1}$ and $M_{2}$ computed for $j=0,1$ and 2 by:

$$
\begin{aligned}
& M_{0}=\frac{2}{2 \pi} \sum_{n=0}^{N-1} P_{A R}(f(n)), \\
& M_{1}=\frac{2}{(2 \pi)^{2}} \sum_{n=0}^{N-1} f(n) P_{A R}(f(n)) \text { and } \\
& M_{2}=\frac{2}{(2 \pi)^{3}} \sum_{n=0}^{N-1}(f(n))^{2} P_{A R}(f(n)) .
\end{aligned}
$$


and W.F. Fincham

(iii) Median frequency (FMED) is the frequency at which the power spectrum is divided into two regions with equal power and can be computed using the formula:

$$
\sum_{n=0}^{F M E D-1} P_{A R}(f(n))=\sum_{F M D D}^{N-1} P_{A R}(f(n)) .
$$

Table A.2 tabulates the statistics of AR spectral measures for the NOR, MYO and MND groups.

\section{A.4 Cepstral Analysis}

The cepstrum of a signal is defined as the inverse Fourier transform of the log power spectrum of the signal. Alternatively, the cepstral coefficients can be derived directly from the AR coefficients using the formulae (Atal, 1976):

$$
\begin{aligned}
& c_{1}=-\alpha_{1} \\
& c_{n}=-\alpha_{n}-\sum_{k=1}^{n-1}(1-k / n) \alpha_{k} c_{n-k} \text { for } 1<n<p
\end{aligned}
$$

and

$$
c_{n}=-\sum_{k=1}^{n-1}(1-k / n), \alpha_{k} c_{n-k} \text { for } n>p
$$

where $\alpha_{n}$ and $c_{n}$ denote the nth $\mathrm{AR}$ and cepstral coefficients respectively. Table A.4 tabulates the statistics of cepstral coefficients $c_{1}$ to $c_{12}$ for the NOR, MYO and MND groups. A more detailed analysis of the AR, spectral and cepstral coefficients estimation analysis is given in Elia (1994).

\section{A.5 Wavelet Analysis}

The Fast Wavelet Transform (FWT) algorithm consists of the decomposition and the reconstruction phases. For our purposes, we focus on describing the decomposition phase as shown in Fig. A.l. In the decomposition phase, the original signal is decomposed into its high 
Volume 8, Nos. 1-2

A Modular Neural Network Decision Support

System in EMG Diagnosis

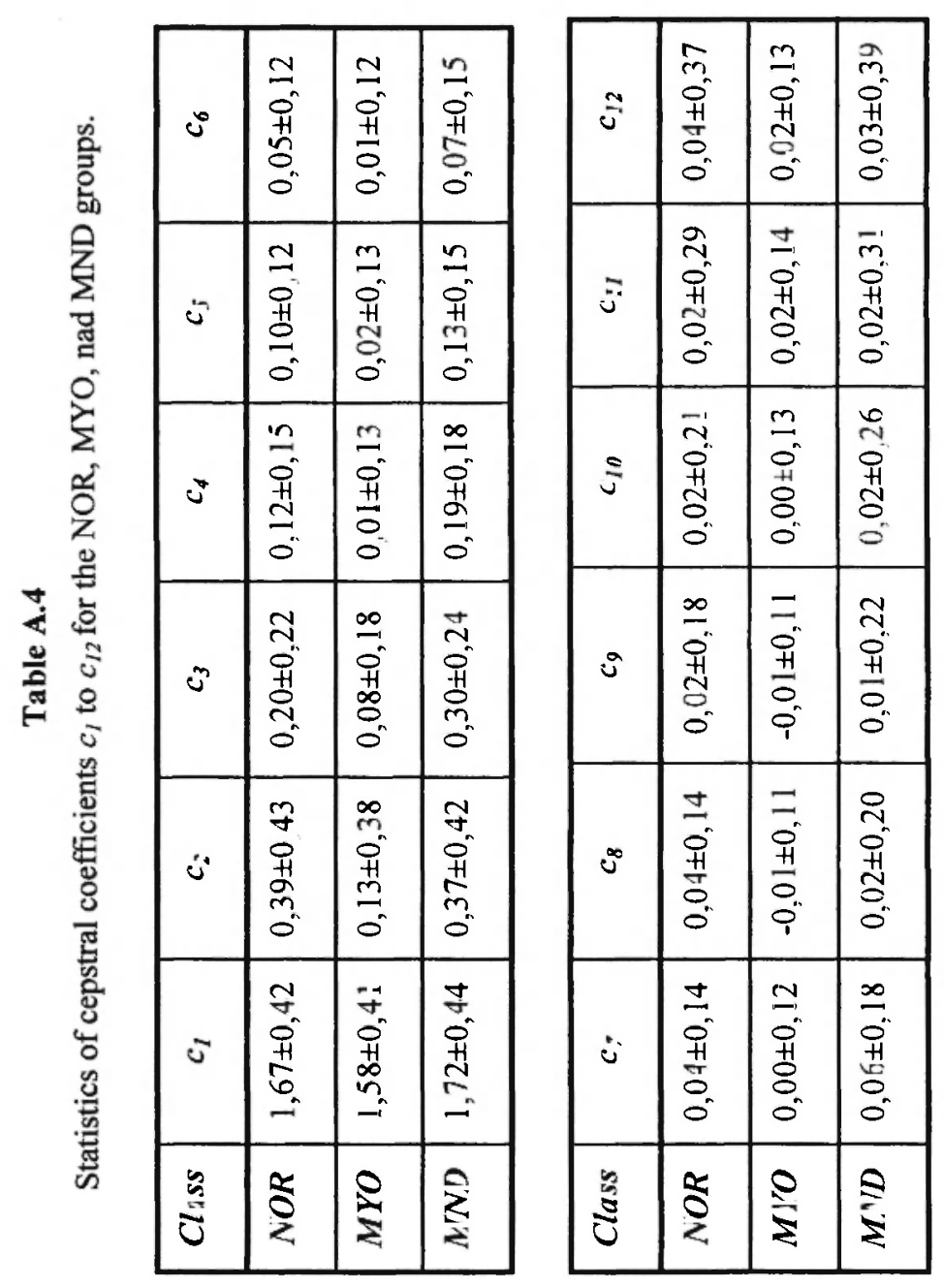


and W.F. Fincham

\section{Decomposition Phase}

Step I: For the first stage, let $\mathrm{s}^{\prime \prime}=\mathrm{s}$ (the input signal).

Step 2: For $j=0$ to $j=\mathrm{k}-1$

$$
\begin{aligned}
& s^{j-1}=\left(s^{\prime *} h\right) \downarrow 2 \\
& d^{j-1}=\left(s^{j * g}\right) \downarrow 2
\end{aligned}
$$

\section{Reconstruction Phase}

Step 3: Using $s^{-k}, d^{-1}, d^{-2}, \ldots . d^{-h}$. reconstruct the input:

$$
\begin{aligned}
& \text { For } j=-k \text { to }-1 \\
& \qquad s^{j+1}=\left\{\left(s^{j}\right) \uparrow 2\right\}^{*} p+\left\{\left(d^{j}\right) \uparrow 2\right\}^{*} q
\end{aligned}
$$

where:
* denotes convolution.
$\downarrow \quad$ denotes down-sampling.
$\uparrow \quad$ denotes up-sampling.
$k \quad$ is the number of stages we want to perform.
$\mathrm{g}, \mathrm{h}$ denote the impulse responses of the decomposition filters.
$p, q$ denote the impulse responses of the reconstruction filters

Fig. A.1: Wavelet transform algorithm: decomposition and reconstruction phases.

frequency and low frequency components. The original discrete signal $x_{0}, x_{1}$, $x_{2}, \ldots$ is low-pass filtered by $h_{0}, h_{1}, h_{2}, \ldots$ and down-sampled by two to produce the low-frequency content signal (smooth signal) $s_{0}^{-1}, s_{1}^{-1}, s_{2}^{-1}, \ldots$ Similarly, the original discrete signal is high-pass filtered by $g_{0}, g_{1}, g_{2}, \ldots$ and down-sampled by two to produce the high-frequency content signal (detail signal) $d_{0}^{-1}, d_{1}^{-1}, d_{2}{ }^{-1}, \ldots$. In our notation, the superscript -1 of $s^{-1}, d^{-1}$ denotes the first decomposition stage. The algorithm is repeated recursively for $j$ stages where for the $j$ th stage, the output signals are denoted by $s^{j}$ and 
$d^{\prime}$. It is important to note that different wavelet transforms can be defined in terms of different sets of decomposition filters. In this study four different wavelets were investigated: Daubechies 4 (DAU4) and 20 (DAU20), Chui $(C H)$ and Battle-Lemarie $(B L)$. For the Daubechies family of wavelets (Daubechies, 1992), the DAU4 is defined in terms of only four coefficients, while the DAU20 is defined in terms of twenty coefficients. Since the DAU4 is affected by much less signal samples than the $D A U 20$, it is clear that the DAU4 has a better time-resolution than the DAU20. On the other hand, by design, the DAU20 provides for a much better approximation to the ideal low-pass/high-pass decomposition filters. This tradeoff between the time and scale resolutions obeys the uncertainty principle. For rapidly-changing signals (like MUAPs), we prefer time-resolution to scale-resolution. From the multi-resolution analysis perspective, the Chui and Battle-Lemarie wavelet transforms provide the familiar piecewise-polynomial approximation to the signal (Chui, 1992). Thus, for these spline-based wavelets, the scalograms measure how much the signal, and its increments jump from point to point (Strang and Nguyen, 1996).

For each MUAP, the MUAP mean was subtracted and each MUAP was normalized to its own power before analysis. MUAP position was adjusted so that the maximum positive peak to occur in the region of 200 points (10ms). Analysis was carried out for six wavelet bands tabulated in table A.5. Table A.6 tabulates the normalized MUAP wavelet power distribution per band per group for $D A U 4, D A U 20, C H$ and $B L$. As it has already been described in section 2.2.5 most of the MUAP power is concentrated in the lower four bands, $d^{-4}, d^{3}, d^{6}$ and $s^{6}$ (Pattichis et al. 1996). A total of 16 coefficients around the main MUAP spike were extracted from each of these bands for the neural network classification.

\section{Acknowledgements}

This work was partly supported by the Cyprus Institute of Neurology and Genetics and the University of Cyprus. 


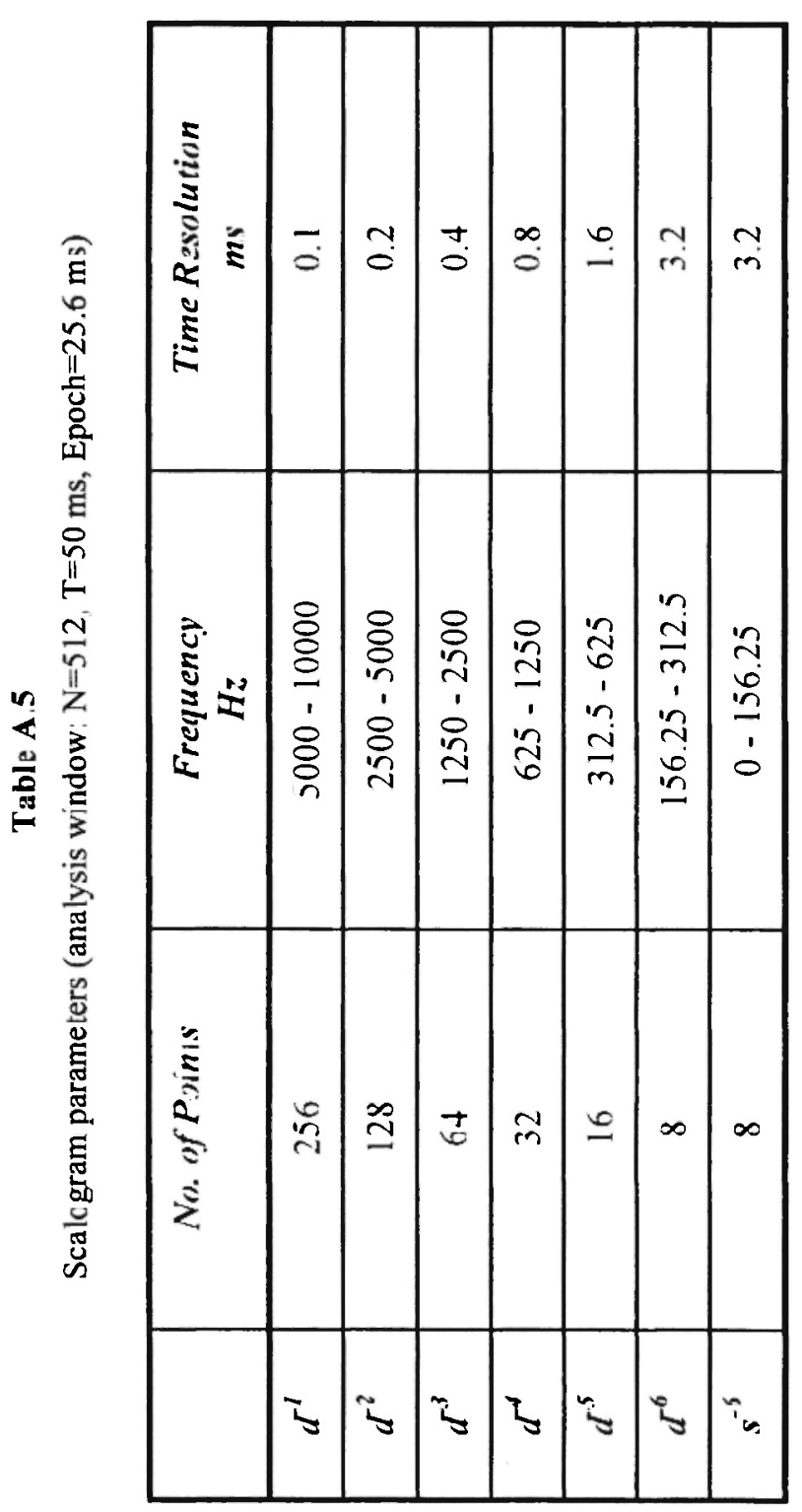




\begin{tabular}{|c|c|c|c|c|c|c|c|c|c|}
\hline \multirow{13}{*}{ 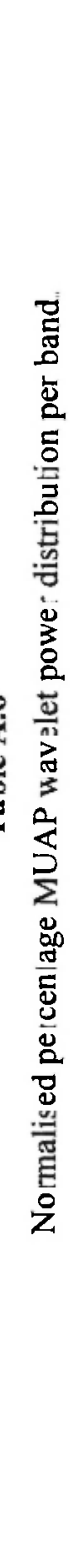 } & \multirow{3}{*}{$\vec{\infty}$} & $\underset{\Sigma}{\Sigma}$ & $\stackrel{0}{0}$ & $\stackrel{\infty}{\stackrel{0}{0}}$ & 满 & $\underset{\infty}{\infty}$ & $\begin{array}{l}0 \\
\infty \\
\approx n\end{array}$ & $\underset{\text { in }}{\stackrel{8}{8}}$ & $\underset{\sim}{\stackrel{\sim}{\sim}}$ \\
\hline & & $\stackrel{s}{\Sigma}$ & $\frac{a}{0}$ & $\stackrel{2}{=}$ & $\begin{array}{l}\mathscr{0} \\
\ddot{I}\end{array}$ & $\begin{array}{l}\stackrel{\sim}{m} \\
\stackrel{\sim}{N}\end{array}$ & $\begin{array}{l}\tilde{N} \\
\stackrel{m}{\rho}\end{array}$ & $\stackrel{m}{\stackrel{m}{I}}$ & $\begin{array}{l}\infty \\
\stackrel{+}{\simeq}\end{array}$ \\
\hline & & $\underset{z}{\xi}$ & $\frac{ \pm}{0}$ & $\stackrel{\infty}{\circ}$ & $\frac{\sim}{\infty}$ & $\stackrel{m}{n}$ & 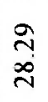 & $\frac{m}{n}$ & $\stackrel{\text { }}{\stackrel{\sim}{2}}$ \\
\hline & \multirow{3}{*}{$\underset{J}{S}$} & $\underset{\xi}{\xi}$ & $\stackrel{\tilde{n}}{\stackrel{\theta}{\theta}}$ & $\frac{\sim}{\sim}$ & $\stackrel{\infty}{2}$ & $\begin{array}{l}\overline{0} \\
\varrho\end{array}$ & $\begin{array}{l}\mathscr{D} \\
\simeq\end{array}$ & $\begin{array}{l} \pm \\
\text { o }\end{array}$ & $\bar{z}$ \\
\hline & & $\stackrel{\Sigma}{\Sigma}$ & $\ddot{\circ}$ & $\stackrel{\infty}{\sim}$ & $\stackrel{c}{\stackrel{c}{\sim}}$ & $\begin{array}{l}\hat{\sigma} \\
\tilde{\sim}\end{array}$ & 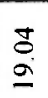 & 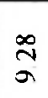 & $\begin{array}{l}\bar{N} \\
\stackrel{\sim}{n}\end{array}$ \\
\hline & & $\frac{\aleph}{\S}$ & $\stackrel{a}{\stackrel{a}{0}}$ & $\stackrel{m}{m}$ & $\begin{array}{l}\infty \\
\stackrel{\infty}{0} \\
\varrho\end{array}$ & $\begin{array}{l}\stackrel{\sim}{\Xi} \\
\underline{\Xi}\end{array}$ & $\begin{array}{l}\vec{\vartheta} \\
\dot{m}\end{array}$ & $\begin{array}{c}\mathcal{O} \\
\text { in }\end{array}$ & $\underset{\mathcal{g}}{\stackrel{f}{f}}$ \\
\hline & \multirow{3}{*}{$\frac{\overparen{S}}{\mathrm{~S}}$} & 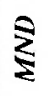 & $\stackrel{0}{0}$ & $\stackrel{0}{\longleftarrow}$ & $\stackrel{g}{a}$ & $\bar{n}$ & 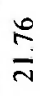 & $\stackrel{m}{m}$ & $\frac{\circ}{\sigma}$ \\
\hline & & $\mathbb{Z}$ & $\frac{\infty}{c}$ & $\stackrel{n}{\stackrel{n}{m}}$ & $\begin{array}{l}\mathfrak{m} \\
\Xi\end{array}$ & $\frac{\infty}{\infty}$ & $\frac{\bar{x}}{m}$ & $\begin{array}{l}\text { 年 } \\
\pm \\
\pm\end{array}$ & $\stackrel{m}{2}$ \\
\hline & & $\frac{\aleph}{z}$ & $\frac{m}{0}$ & $\frac{ \pm}{i}$ & $\stackrel{\text { }}{\sim}$ & $\begin{array}{l}\stackrel{\sim}{0} \\
\stackrel{\varrho}{0}\end{array}$ & $\begin{array}{l}\stackrel{\infty}{\dot{d}} \\
\stackrel{\sim}{\sim}\end{array}$ & $\frac{\infty}{2}$ & 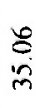 \\
\hline & \multirow{3}{*}{$\frac{\vec{J}}{\mathrm{~K}}$} & 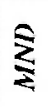 & $\stackrel{m}{0}$ & $\stackrel{a}{=}$ & $\underset{\sim}{\sim}$ & 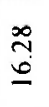 & $\underline{m}$ & $\frac{m}{n}$ & $\underset{\substack{\infty \\
\text { in }}}{\bar{n}}$ \\
\hline & & $\mathbb{Z}$ & $\stackrel{\circ}{\circ}$ & $\underset{m}{E}$ & $\underset{\infty}{\stackrel{N}{*}}$ & $\begin{array}{l}\stackrel{\infty}{\sim} \\
\underset{\mathcal{F}}{\mathcal{H}}\end{array}$ & $\stackrel{n}{\stackrel{n}{\sim}}$ & 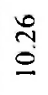 & $\begin{array}{l}\infty \\
m \\
i n\end{array}$ \\
\hline & & $\frac{\nwarrow}{\xi}$ & مٌ & $\stackrel{R}{2}$ & $\stackrel{0}{n}$ & 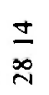 & $\begin{array}{l}\mathfrak{n} \\
=\end{array}$ & $\underset{q}{\tilde{g}}$ & $\stackrel{m}{m}$ \\
\hline & & & $y$ & 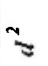 & $r$ & $z$ & $y$ & $\stackrel{\circ}{\longrightarrow}$ & in \\
\hline
\end{tabular}


and W.F. Fincham

\section{References}

Akaike H., 1974, "Power spectrum estimation through autoregression model fitting", Ann. Inst. Stat. Math., Vol 21, pp. 407-419.

Andreassen S., Jensen F.V., Andersen S.K., Falck B., Kjaerulff U., Woldbye M. Soerensen A.R, Rosenfalck A., Jensen F., 1989, "MUNIN - an expert EMG assistant", Computer-Aided Electromyography and Expert systems, ed. by J.E. Desmedt, Elsevier Science Publishers, pp. 255-277.

Atal B. S., 1976, "Automatic recognition of speakers from their voices", IEEE Proc., Vol 64, No 4, pp. 460-475.

Blinowska K.J., Hausmanowa-Petrusewich I., Miller-Larson A., Zachara J., 1980, "The Analysis of simple EMG potentials by means of multivariate methods", Electromyogr، Clin. Neurophysiol., Vol. 20, pp. 105-123.

Box G.E.P., Jenkins G.M., 1976, Time Series Analysis: Forecasting and Control, 2nd edition, Holden-Day, San Francisco CA.

Broomhead D.S., Lowe D., 1988, "Multivariable functional interpolation and adaptive networks", Complex Systems 2, pp. 321-355.

Buchthal F. 1957, An Introduction to Electromyography, Copenhagen, Gyldendal.

Chen S., Cowan C.F.N, Grant P.M, 1991, "Orthogonal Least Squares Learning Algorithms for Radial Basis Function Networks", IEEE Trans. on Neural Networks, Vol. 2, No 2, pp. 302-309, March, 1991.

Christodoulou C.I., Pattichis C.S., 1995, "A New Technique for the Classification and Decomposition of EMG signals", in Proc. IEEE International Conference on Neural Networks, Perth, Western Australia, Vol. 5, pp. 2303-2308, Nov. 1995.

Chui C.K., 1992, Wavelet Analysis and its Applications, Volume 1. An Introduction to Wavelets, San Diego: Academic Press.

Coatrieux J.L., Toulouse P., Rouvrais B., Bars R.L., 1983, "Automatic classification of electromyographic signals", EEG Clin. Neurophysiol, Vol. 55, pp. 333-341.

Daubechies I., 1992, "Ten Lectures On Wavelets", Philadelphia, Pensylvania: SLAM. 
Demuth H, Beale M. 1994, MATLAB, Neural Network Toolbox User's Guide, The MathsWork, Inc., Natick, MA.

Elia A. G., 1994, "Autoregressive Spectral Modelling of Motor Unit Action Potentials", M.Phil. thesis, QMW College, University of London.

France F.R, Santucci G.S.(eds), 1991, Perspectives of Information Processing in Medical Applications, Springer Verlag.

Fuglsang-Frederiksen A., Jeppesen S.M., 1989, "A rule-based expert system for diagnosing neuromuscular disorders", Computer-Aided Electromyography and Expert systems, ed. by J.E. Desmedt, Elsevier Science Publishers, pp. 289-296.

Gallant S.I, 1995, "Expert Systems and Decision Systems using Neural Networks", The Handbook of Brain Theory and Neural Networks, ed. by M.A. Arbib, MIT" Press, Cambridge, Massachusetts, pp. 377-380.

Haykin S., 1994, Neural Networks - A comprehensive foundation, Macmillan College Publishing Company.

Jacobs R. A., Jordan M. I., 1991, "A competitive modular connectionist architecture", Advances in Neural Information Processing Systems, ed. by R.P. Lippmann, J.E. Moody and D.J. Touretzky, San Mateo, CA: Morgan Kaufinann, pp. 767-773.

Jordan M. I., Jacobs R. A., 1995, "Modular and Hierarchical Learning Systems", The Handbook of Brain Theory and Neural Networks, ed. by M.A. Arbib, MIT Press, Cāmbridge, Massachusetts, pp. 186-234.

Kohonen T., 1990, "The Self-Organizing Map", Proceedings of the IEEE, Vol. 78, No. 9, pp. 1464-1480, Sept. 1990.

Kohonen T., 1995, Self-Organizing Maps, Springer Series in Information Sciences, Berlin.

Lindstrom L., Petersen I., 1983, "Power spectrum analysis of EMG signals and its applications", Prog. Clin. Neurophysiol., Vol.10, ed. by J.E. Desmedt, pp. 1-51, 1983.

Marple S. L. Jr., 1987, Digital Spectral Analysis with Applications, Prentice-Hall, USA.

Pattichis C.S., 1992, "Artificial neural networks in clinical electromyography", Ph.D. dissertation, $Q M W$ College, University of London. 
and W.F. Fincham

Pattichis C.S., Schizas C.N., Mittleton L.T., 1995, "Neural Network Models in EMG diagnosis", IEEE Trans. on Biomedical Engineering, Vol. 42, No 5, pp. 486-496, May 1995.

Pattichis C.S., Schizas C.N., 1996, "Genetics-Based Machine Learning for the Assessment of Certain Neuromuscular Disorders", IEEE Trans. on Neural Networks, Vol. 7, No 2, pp. 427-439, March 1996.

Pattichis C.S., Pattichis M.S., Schizas C.N., 1996, "MUAP Wavelet Analysis", Proceedings of the 18th Annual International Conference of the IEEE Engineering in Medicine and Biology, paper no. 553, Amsterdam, Netherlands.

Rioul O., Vetterli M., 1991, "Wavelets and signal processing", IEEE SP magazine, pp. 14-38.

Rosenfalck P., Rosenfalck A, 1975, "Electromyography - sensory and motor conduction. Findings in normal subjects", Copenhagen: Laboratory of Clinical Neurophysiology, Rhigshospitalet, pp. 1-49.

Rumelhart D.E., Hinton G.E., Williams R.J., 1986, "Learning internal representations by error propagation", Parallel Distributed Processing, Vol.1, ed. by D.E. Rumelhart and J.L. McClelland, pp. 318-362.

Schizas C.N., Pattichis C.S., Bonsett C.A., 1994, "Medical diagnostic systems: a case for neural networks", Technology and Health Care, 2, pp. 1-18.

Schofield I.S., 1997, "HINT - High Level Inferencing Tool: An expert system for the interpretation of neurophysiological studies", Journal of Intelligent Systems, special issue on Computational Intelligent Diagnostic Systems in Medicine, ed. by C. Schizas, this volume.

Stalberg E., Andreassen S., Falck B., Lang H., Rosenfalck A., Trojaborg W. (1986), "Quantitative analysis of individual motor unit potentials: A proposition for standardised terminology and criteria for measurement", $J$. Clin. Neurophysiol., Vol. 3, No. 4, pp. 313-348.

Stalberg E., Bishoff C., Falck B., 1994, "Outliers, a way to detect abnormality in quantitative EMG", Muscle and Nerve, Vol. 17, pp. 392-399, April 1994.

Strang G., Nguyen T., 1996, Wavelets and Filter Banks, WellesleyCambridge Press, Wellesley, MA.

Tumer K., Ghosh J., 1996, "Theoretical foundations of linear and order statistics combiners for neural pattem classifiers", Dept. of Electrical and Computer Engineering, University of Texas, Austin. 
Brought to you by | provisional account

Authenticated

Download Date | 1/22/19 2:52 PM 